\title{
Dynamic Iterative Reconstruction for Interventional 4-D C-Arm CT Perfusion Imaging
}

\author{
Michael T. Manhart, IEEE Graduate Student Member, Markus Kowarschik, Andreas Fieselmann, Yu \\ Deuerling-Zheng, Kevin Royalty, Andreas K. Maier and Joachim Hornegger, IEEE Member
}

\begin{abstract}
Tissue perfusion measurement using C-arm angiography systems capable of CT-like imaging (C-arm CT) is a novel technique with potentially high benefit for catheter guided treatment of stroke in the interventional suite. However, perfusion $\mathrm{C}$-arm CT (PCCT) is challenging: the slow $\mathrm{C}$-arm rotation speed only allows measuring samples of contrast time attenuation curves (TACs) every 5-6 s if reconstruction algorithms for static data are used. Furthermore, the peak values of the TACs in brain tissue typically lie in a range of $5-30 \mathrm{HU}$, thus perfusion imaging is very sensitive to noise. We present a dynamic, iterative reconstruction (DIR) approach to reconstruct TACs described by a weighted sum of basis functions. To reduce noise, a regularization technique based on joint bilateral filtering (JBF) is introduced. We evaluated the algorithm with a digital dynamic brain phantom and with data from six canine stroke models. With our dynamic approach, we achieve an average Pearson correlation (PC) of the PCCT canine blood flow maps to coregistered perfusion $C T$ maps of 0.73 . This $P C$ is just as high as the $\mathrm{PC}$ achieved in a recent PCCT study, which required repeated injections and acquisitions.
\end{abstract}

Index Terms-Perfusion imaging, dynamic reconstruction, Carm CT, stroke treatment

M. Manhart, A. Fieselmann, A. Maier and J. Hornegger are with Pattern Recognition Lab, Department of Computer Science, Friedrich-Alexander-Universität Erlangen-Nürnberg, Martensstr. 3, 91058 Erlangen, Germany. M. Kowarschik and Y. Deuerling-Zheng are with Siemens AG, Angiography \& Interventional X-Ray Systems, Forchheim, Germany. A. Fieselmann and J. Hornegger are with Erlangen Graduate School in Advanced Optical Technologies (SAOT). K. Royalty is with Siemens Medical Solutions, Malvern, PA, USA, and Department of Biomedical Engineering, University of Wisconsin, Madison, WI, USA. Email: michael.manhart@cs.fau.de ${ }^{1}$

\section{INTRODUCTION}

Perfusion CT (PCT) is an important imaging modality for diagnosis in case of an ischemic stroke event. Time attenuation curves (TACs) of contrast flow in tissue and vessels are extracted from a time series of brain volumes acquired after a contrast bolus injection. Perfusion parameter maps calculated from TACs, which represent quantities such as cerebral blood flow $(\mathrm{CBF})$, cerebral blood volume $(\mathrm{CBV})$, mean transit time (MTT), and time-to-peak (TTP), provide information about the extent of the affected tissue [1]. They can be used to identify potentially salvageable ischemic tissue that may be reperfused by catheter-guided stroke therapy procedures such

\footnotetext{
${ }^{1}$ Copyright (c) 2013 IEEE. Personal use of this material is permitted. However, permission to use this material for any other purposes must be obtained from the IEEE by sending a request to pubs-permissions@ieee.org.
}

as intra-arterial thrombolysis [2]. For this purpose, the patient is transported to an interventional suite equipped with a $\mathrm{C}$-arm angiography system where perfusion measurement is not yet available. Perfusion measurement using $\mathrm{C}$-arm systems would allow the assessment of the perfusion parameters directly before and during the interventional procedure and thus help to determine treatment success and endpoint. Current $\mathrm{C}$-arm systems typically require $\sim 4-5 \mathrm{~s}$ to acquire the $\mathrm{X}$-ray projection images needed to reconstruct one volume and a pause of $\sim 1 \mathrm{~s}$ between two successive acquisitions, which limits the temporal sampling of the TACs and makes perfusion C-arm CT (PCCT) challenging.

Recently, new approaches have been presented to overcome these problems: an acquisition protocol combining interleaved scanning and partial reconstruction interpolation has been presented and evaluated in [3] and [4]. While providing improved temporal sampling and high computational efficiency, multiple scanning sequences are required, which increases irradiation and contrast agent dose to the patient. Serowy et. al [5] and Neukirchen et al. [6] presented iterative modelbased approaches, which reconstruct TACs described by a weighted sum of smooth temporal basis functions in order to keep the degrees of freedom relatively low. Although these algorithms can be implemented similarly to classical algebraic reconstruction techniques (ART) [7], the use of basis functions with non-compact support introduces additional computationally expensive steps. A detailed survey on existing techniques for PCCT is given in [8].

Based on the iterative parameter optimization algorithm by Neukirchen et al. [6], we introduced a graphics processing unit (GPU) based dynamic iterative reconstruction (DIR) [9] algorithm using linear basis functions with compact support in combination with a denosing strategy based on joint bilateral filtering (JBF) [10]. In this work, we additionally describe this algorithm in more detail, include the noise statistics by statistical ray weighting, and evaluate the use of different basis functions to model the reconstructed TACs by numerical simulations. Furthermore, we validate our methods more extensively using in vivo data from six canines with induced ischemic stroke and also include MTT and TTP perfusion parameters in the evaluation.

Our paper is organized as follows. In Section II, we describe our C-arm CT perfusion imaging acquisition protocol. The mathematical formulation and the implementation details of our dynamic reconstruction approach are presented in Section III. We will investigate our algorithm in Section IV using simulated data and real data from an in vivo study in a canine 
stroke model. The results of the evaluation and the benefits of the dynamic reconstruction approach are discussed in Section V. In Section VI, we summarize and conclude our work.

\section{Perfusion C-Arm CT Scanning Protocol}

This section describes the $\mathrm{C}$-arm perfusion acquisition protocol used for the simulation studies and real data acquisitions. Since currently available C-arm systems are not capable of continuous, uni-directional rotations, the $\mathrm{C}$-arm is rotated in a bi-directional manner in forward and backward direction. At first, one C-arm rotation in forward and one in backward direction acquire mask projections with the static anatomical structures. Mask projections in both directions need to be acquired because the X-ray source and detector positions differ slightly during the forward and backward rotations. In each rotation, $N_{\text {proj }}=248$ projections along an angular range of $197.6^{\circ}$ are acquired. After contrast agent injection, the Carm is rotated $N_{\text {rot }}=7$ times in bi-directional manner as illustrated in Figure 1. Each rotation takes $T_{\text {rot }}=4.3 \mathrm{~s}$, with a pause of $T_{\text {stop }}=1.2 \mathrm{~s}$ between two successive rotations. Thus, direct reconstruction of the rotations would allow a temporal sampling of TACs with period $T_{\mathrm{s}}=T_{\text {rot }}+T_{\text {stop }}=5.5 \mathrm{~s}$ during the total scan time $T_{\text {scan }}=N_{\text {rot }} \cdot T_{\text {rot }}+\left(N_{\text {rot }}-1\right) \cdot T_{\text {stop }}=37.3 \mathrm{~s}$.

We denote the measured photon counts (X-ray intensities) of all $N_{\text {rot }}$ contrast-enhanced sweeps by vector $\mathbf{k}^{\mathrm{B}} \in \mathcal{N}^{S_{\mathrm{P}} \cdot N_{\mathrm{P}}}$ (B: bolus), where $S_{\mathrm{P}}$ denotes the number of detector pixels and $N_{\mathrm{P}}=N_{\text {rot }} \cdot N_{\text {proj }}$ the total number of contrast-enhanced projections. The mask photon counts are denoted by the vector $\mathbf{k}^{\mathrm{M}} \in \mathcal{N}^{S_{\mathrm{P}} \cdot N_{\mathrm{P}}}$ (M: mask). Each entry in vector $\mathbf{k}^{\mathrm{M}}$ is given by the intensity value of the mask projection, which corresponds to the intensity value of the respective contrastenhanced projection in vector $k^{B}$ (i.e., with the same source position and ray direction). By logarithmic pre-processing of $\mathbf{k}^{\mathrm{M}}$ and $\mathbf{k}^{\mathrm{B}}$, the $\mathrm{X}$-ray attenuation line integrals denoted by the vectors $\mathbf{p}^{\mathrm{M}}$ and $\mathbf{p}^{\mathrm{B}}$ are computed:

$$
p_{i}^{\mathrm{M} / \mathrm{B}}=\ln \frac{k_{i}^{\mathrm{S}}}{k_{i}^{\mathrm{M} / \mathrm{B}}}, \quad i=1 \ldots S_{\mathrm{P}} \cdot N_{\mathrm{P}},
$$

where $k_{i}^{\mathrm{S}}$ denotes the number of emitted photons for ray $i$. The mask projections are subtracted from the contrastenhanced projections to generate the projection data vector $\mathbf{p}=\mathbf{p}^{\mathrm{B}}-\mathbf{p}^{\mathrm{M}}=\left[\mathbf{p}_{1}^{T} \cdots \mathbf{p}_{N_{\mathrm{P}}}^{T}\right]^{T}, \mathbf{p} \in \mathcal{R}^{S_{\mathrm{P}} \cdot N_{\mathrm{P}}}$ containing the line integrals describing the pure contrast dynamics and noise. Furthermore, the vector $\mathbf{t}^{\mathrm{P}}=\left[t_{1}^{\mathrm{P}} \cdots t_{N_{\mathrm{P}}}^{\mathrm{P}}\right]$ describes the acquisition time points of every projection in $\mathbf{p}$. Table I shows an overview of all acquisition parameters.

\section{Dynamic Reconstruction Algorithm}

\section{A. Mathematical Formulation}

Modeling of Time Attenuation Curves: There is a continuous contrast flow during the acquisition, so the observed volume is different for each of the $N_{\mathrm{P}}$ projection images. For an exact solution, we would have to reconstruct the $4 \mathrm{D}$ volume vector $\mathbf{x}=\left[\mathbf{x}_{1}^{T} \cdots \mathbf{x}_{N_{\mathrm{P}}}^{T}\right]^{T}$, consisting of $N_{\mathrm{P}} 3 \mathrm{D}$ volumes $X_{i} \in \mathcal{R}^{N_{\mathrm{x}} \times N_{\mathrm{y}} \times N_{z}}$ represented as column vectors $\mathbf{x}_{i} \in \mathcal{R}^{S_{\mathrm{V}}}$ with $S_{\mathrm{V}}=N_{\mathrm{x}} \cdot N_{\mathrm{y}} \cdot N_{\mathrm{z}}$ and $i=1 \ldots N_{\mathrm{P}}$. To describe the mapping of the $4 \mathrm{D}$ volume to the projection data, we define

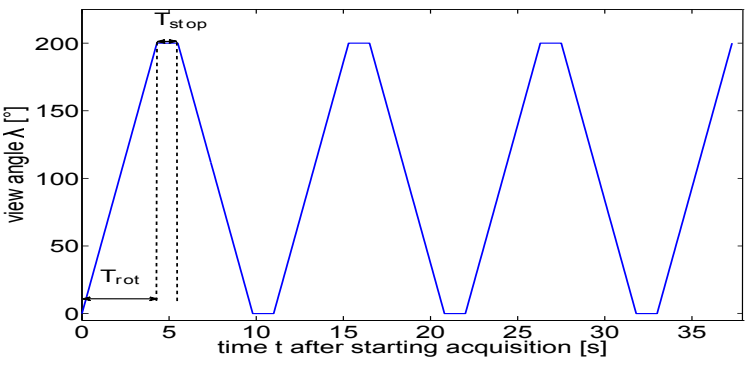

Figure 1: C-arm acquisition protocol.

\begin{tabular}{cc}
\hline view-angle increment & $0.8^{\circ}$ \\
number of views per rotation $\left(N_{\text {proj }}\right)$ & 248 \\
angular range per rotation & $197.6^{\circ}$ \\
time per rotation $\left(T_{\text {rot }}\right)$ & $4.3 \mathrm{~s}$ \\
time between rotations $\left(T_{\text {stop }}\right)$ & $1.2 \mathrm{~s}$ \\
number of rotations $\left(N_{\text {rot }}\right)$ & 7 \\
total scanning time $\left(T_{\text {scan }}\right)$ & $37.3 \mathrm{~s}$ \\
source-to-detector distance & $1200 \mathrm{~mm}$ \\
detector pixel size & $0.616 \times 0.616 \mathrm{~mm}^{2}$ \\
number of detector pixels $\left(S_{\mathrm{P}}\right)$ & $616 \times 480$ \\
total detector size & after $4 \times 4 \mathrm{rebinning}$ \\
tube peak voltage & $\approx 380 \times 296 \mathrm{~mm}^{2}$ \\
system dose & $70 \mathrm{kVp}$ \\
\hline
\end{tabular}

Table I: Acquisition parameters.

the system matrix $A$ assembled from matrices $A_{i}$, which map the $3 \mathrm{D}$ volumes to the projection images according to the acquisition geometry, such that $\mathbf{p}_{i}=A_{i} \mathbf{x}_{i}$ and $\mathbf{p}=A \mathbf{x}$ :

$$
A=\left(\begin{array}{cccc}
A_{1} & \mathbf{0} & \cdots & \mathbf{0} \\
\mathbf{0} & A_{2} & \ddots & \mathbf{0} \\
\vdots & \vdots & \ddots & \vdots \\
\mathbf{0} & \mathbf{0} & \cdots & A_{N_{\mathrm{P}}}
\end{array}\right)
$$

with $A_{i} \in \mathcal{R}^{S_{\mathrm{P}} \times S_{\mathrm{V}}}$ and $A \in \mathcal{R}^{\left(N_{\mathrm{P}} \cdot S_{\mathrm{P}}\right) \times\left(N_{\mathrm{P}} \cdot S_{\mathrm{V}}\right)}$. Directly solving $\mathbf{p}=A \mathbf{x}$ for the exact solution $\mathbf{x}$ is not possible since the equation system is heavily underdetermined. Therefore we constrain each of the TACs described by $\mathbf{x}$ to be inside a subspace spanned by a set of $N_{\mathrm{w}}$ basis functions $b_{j}(t), j=1 \ldots N_{\mathrm{w}}$, such that the vector $\mathbf{x}_{i}$ defining the contrast attenuation in the volume at time point $t_{i}^{\mathrm{P}}$ is computed by a linear combination of basis functions with weight vectors $\mathbf{w}_{j}$ :

$$
\mathbf{x}_{i}=\sum_{j=1}^{N_{\mathrm{w}}} b_{j}\left(t_{i}^{\mathrm{P}}\right) \mathbf{w}_{j}, \quad \mathbf{w}_{j} \in \mathcal{R}^{S_{V}} .
$$

The interpolation of the 4D volume vector $\mathrm{x}$ from all basis weights $\mathbf{w}=\left[\mathbf{w}_{1}^{T} \cdots \mathbf{w}_{N_{\mathrm{w}}}^{T}\right]^{T}$ is a linear operation denoted by the matrix $B \in \mathcal{R}^{\left(N_{\mathrm{P}} \cdot S_{\mathrm{V}}\right) \times\left(N_{\mathrm{w}} \cdot S_{\mathrm{V}}\right)}$ such that $\mathbf{x}=B \mathbf{w}$. Using basis functions to describe the TACs reduces the degrees of freedom of the reconstruction problem from $S_{\mathrm{V}} \cdot N_{\mathrm{P}}$ to $S_{\mathrm{V}} \cdot N_{\mathrm{w}}$. To reconstruct the basis weights $\tilde{\mathrm{w}}$ from the measured projection data $\mathbf{p}$, one needs to solve the least-squares problem minimizing the Euclidean distance between the measured projection data $\mathbf{p}$ and the forward projected estimated $4 \mathrm{D}$ 
volume:

$$
\tilde{\mathbf{w}}=\frac{1}{2} \underset{\mathbf{w}}{\operatorname{argmin}}\|A B \mathbf{w}-\mathbf{p}\|_{2}^{2} .
$$

In this optimization problem the weight vector $\mathbf{w}$ and the interpolation matrix $B$ describe the temporal dynamics of the contrast flow and the matrix $A$ describes the projection geometry of the C-arm system.

Statistical Ray Weighting: In the next step we include a statistical noise model into Equation 4 to transform this equation into the maximum likelihood (ML) estimate of the weights $\check{\text { w }}$ from projection data corrupted by quantum noise. To account for quantum noise, the numbers of measured photons can be described by independent Poisson random processes [11].

First, we discuss how we model the noise in the projection data $\mathbf{p}$, which is generated by logarithmic pre-processing of $\mathbf{k}^{\mathrm{M}}$ and $\mathbf{k}^{\mathrm{B}}$ followed by subtraction. Let $\hat{k} \sim \mathcal{P}(\mu=\bar{k})$ be a Poisson random variable describing a photon measurement with mean $\bar{k}$ corresponding to the unknown number of ideally measured arriving photons. Since we do tomographic brain imaging we assume a large number of counts; i.e., $\bar{k}>1000$. For such large counts, a Gaussian process is an excellent approximation of the Poisson process:

$$
\hat{k} \sim \mathcal{N}\left(\mu=\bar{k} ; \sigma^{2}=\bar{k}\right) .
$$

By taking the logarithm of $\hat{k}$ according to Equation 1, we obtain the random variable $\hat{p}$ describing the corresponding line integral. The distribution of $\hat{p}$ can be approximated according to [12] by:

$$
\hat{p} \sim \mathcal{N}\left(\mu=\bar{p}=\ln \left(k^{\mathrm{S}} / \bar{k}\right) ; \sigma^{2}=1 / \bar{k}\right) .
$$

If $\hat{p}^{\mathrm{B}}$ and $\hat{p}^{\mathrm{M}}$ denote contrast-enhanced and corresponding mask measurements, respectively, which are two independent Gaussian random variables, their difference $\hat{p}^{\mathrm{S}}=\hat{p}^{\mathrm{B}}-\hat{p}^{\mathrm{M}}$, which represents the pure contrast enhancement, is also a Gaussian random variable:

$$
\hat{p}^{\mathrm{S}} \sim \mathcal{N}\left(\mu=\bar{p}^{\mathrm{B}}-\bar{p}^{\mathrm{M}} ; \sigma^{2}=1 / \bar{k}^{\mathrm{B}}+1 / \bar{k}^{\mathrm{M}}\right) .
$$

Thus, the noise in the subtracted projection data $\mathbf{p}$ can be modeled by additive Gaussian noise, where the variance is related to the number of photon counts measured in mask and bolus acquisitions. Since the contrast attenuation is very small compared to the attenuation of the anatomic structures, we approximate the variance $\sigma_{i}$ of each entry $p_{i}$ in $\mathbf{p}$ by the number of photons measured in the corresponding mask acquisition:

$$
\sigma_{i}^{2} \approx 1 / k_{i}^{\mathrm{B}}+1 / k_{i}^{\mathrm{M}} \approx 2 / k_{i}^{\mathrm{M}} .
$$

The maximum likelihood (ML) estimation of the weights $\check{\mathbf{w}}$ from projection data with Gaussian noise is provided by the weighted least squares function $\mathcal{D}(\mathbf{w})$ [13], which combines Equation 4 with the diagonal weighting matrix $D$ :

$$
\begin{aligned}
\check{\mathbf{w}} & =\underset{\mathbf{w}}{\operatorname{argmin}} \mathcal{D}(\mathbf{w}), \\
\mathcal{D}(\mathbf{w}) & =\frac{1}{2}(A B \mathbf{w}-\mathbf{p})^{T} D(A B \mathbf{w}-\mathbf{p}),
\end{aligned}
$$

where $D=\operatorname{diag}\left\{k_{1}^{\mathrm{M}} / 2, \cdots, k_{S_{\mathrm{P}} \cdot N_{\mathrm{P}}}^{\mathrm{M}} / 2\right\}$.
Landweber Iteration: It is practically infeasible to solve Equation 9 directly, because the data dimensions are huge and the system matrix of image reconstruction problems is typically ill-conditioned [14]. We solve this large scale problem as described in [6] by a gradient-based iterative procedure using the Landweber iteration scheme [15]:

$$
\mathbf{w}^{\text {new }}=\mathbf{w}^{\text {old }}+\beta \cdot B^{T} A^{T} D\left(\mathbf{p}-A B \mathbf{w}^{\text {old }}\right) .
$$

The parameter $\beta$ controls the step size of the parameter update in each iteration. The matrix product $A B$ describes the calculation of the $4 \mathrm{D}$ volume $\mathrm{x}$ by interpolation using the basis functions followed by the forward projection using the system matrix $A$. The diagonal matrix $D$ weights each entry of the error image $\mathbf{p}-A B \mathbf{w}^{\text {old }}$ according to its statistical uncertainty resulting in the weighted error vector $\mathbf{e}=D\left(\mathbf{p}-A B \mathbf{w}^{\text {old }}\right)$. The matrix product $B^{T} A^{T}$ is a back projection of $\mathbf{e}$ into the weight vector $\mathbf{w}^{\text {old }}$, where $B^{T}$ introduces an additional weighting of the back projected errors.

To describe the effect of the transposed interpolation matrix $B^{T}$, we split the weighted error vector $\mathbf{e}$ into parts $\mathbf{e}_{i}, i=$ $1 \ldots N_{\mathrm{P}}$, where $\mathbf{e}_{i}$ corresponds to projection image $\mathbf{p}_{i}$. Now we can define the update of the weight vector $\mathbf{w}_{j}$ belonging to basis function $b_{j}(t)$ by:

$$
\mathbf{w}_{j}^{\text {new }}=\mathbf{w}_{j}^{\text {old }}+\beta \cdot \sum_{i=1}^{N_{\mathrm{P}}} b_{j}\left(t_{i}^{\mathrm{P}}\right) A_{i}^{T} \mathbf{e}_{i}^{k} .
$$

Vessel-Masked Back Projection: Figure 2a shows a slice of the temporal maximum intensity projection (MIP) volume reconstructed from digital brain phantom data (see Section IV-A1 for details) after 30 iterations of the Landweber scheme according to Equation 11 (with $D=\mathbf{I}$ since data without noise was used). Severe streaking artifacts around the vessels show up. The MIP volume is created from the peak of each TAC. To avoid these artifacts, we apply an empirical modification to the weight update step in Equation 11 by using a vessel-masked back projection. The back projection step is modified such that rays intersecting with high contrast vessel structures are only used for updating voxels containing vessels. Therefore, a first reconstruction of the projection data $\mathbf{p}$ is done using a standard FDK reconstruction method [16] to initialize the weights $\mathbf{w}$. Then, the temporal MIP volume M is computed and a binary vessel mask $\mathbf{v}^{\mathrm{V}} \in\{0,1\}^{S_{\mathrm{V}}}$ in volume space is created by thresholding M. By forward projection of $\mathbf{v}^{\mathrm{V}}$ a vessel mask in projection space $\mathbf{v}^{\mathrm{P}} \in\{0,1\}^{S_{\mathrm{P}} \cdot N_{\mathrm{P}}}$ is calculated (Figure 2c). The complete error image $\mathbf{e}$ is only back projected into vessel voxels indicated by $\mathbf{v}^{\mathrm{V}}$, into the remaining voxels (indicated by $\neg \mathbf{v}^{\mathrm{V}}$ ) only the rays without any vessel intersection (indicated by $\neg \mathbf{v}^{\mathrm{P}}$ ) are back projected ( $\otimes$ describes element-wise multiplication and $\neg$ describes element-wise negation):

$$
\begin{aligned}
\mathbf{w}^{\text {new }} & =\mathbf{w}^{\text {old }}+\beta \cdot B^{T} \triangle \mathbf{w}_{\mathrm{V}}, \\
\triangle \mathbf{w}_{\mathrm{V}} & =\mathbf{v}^{\mathrm{V}} \otimes A^{T} \mathbf{e}+\neg \mathbf{v}^{\mathrm{V}} \otimes A^{T}\left(\neg \mathbf{v}^{\mathrm{P}} \otimes \mathbf{e}\right) .
\end{aligned}
$$

With the modified back projection step, the streaking artifacts are avoided as shown in the MIP volume in Figure 2b. Details on the initialization and the vessel mask creation are given in subsection III-C. 

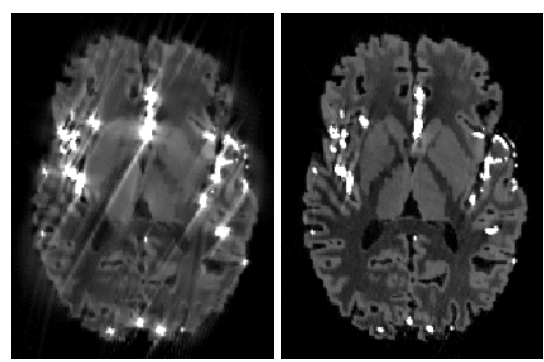

(a) w/o vessel masking (b) w/ vessel masking

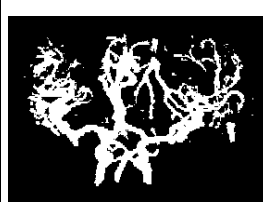

(c) projection mask
Figure 2: Maximum intensity projection (MIP) slices of a 4D perfusion volume reconstructed without and with vesselmasked back projection (window: [0 50] HU).

Bayesian Regularization by Joint Bilateral Filtering: Perfusion imaging is highly sensitive to noise. To allow reliable reconstruction of TACs under noisy conditions, we use joint bilateral filtering for regularization of the DIR results. Bilateral filtering [17], [18] is a non-linear, edge-preserving noise filter using a combination of domain and range filtering. In joint bilateral filtering (JBF), a guidance image is used to calculate the range similarity [10]. In our approach, we use the peak value of each reconstructed TAC to identify voxels with similar TACs (such as vessels or healthy and stroke-affected tissue). This results in a bilateral filter, where the range similarity measure is calculated using the temporal MIP M as guidance image instead of the filtered volume itself. The temporal MIP $M$ is defined as (max describes row-wise maximum selection):

$$
\mathbf{M}=\max \left\{\mathbf{x}_{k} \mid k=1 \ldots N_{\mathrm{w}}\right\}, \quad \mathbf{x}_{k}=\sum_{j=1}^{N_{\mathrm{w}}} b_{j}\left(t_{k}^{\mathrm{w}}\right) \mathbf{w}_{j},
$$

where $t_{k}^{\mathrm{w}}, k=1 \ldots N_{\mathrm{w}}$, denotes the temporal position of the spline knot of the basis function with index $k$.

Since the JBF operation is a 3D filter, we change the notation of the weight vectors for defining the filter. The $3 \mathrm{D}$ volume representation of weight vector $\mathbf{w}_{j}$ is denoted by $W_{j} \in \mathcal{R}^{N_{\mathrm{x}} \times N_{\mathrm{y}} \times N_{\mathrm{z}}}$, and $W_{j}(\mathbf{i})$ is the weight value at $3 \mathrm{D}$ voxel index $\mathbf{i}=\left(i_{x}, i_{y}, i_{z}\right) \in \mathcal{N}^{3}$. The filtered volumes, denoted by $W_{j}^{\mathrm{JBF}}, j=1 \ldots N_{\mathrm{W}}$, are computed by:

$$
\begin{aligned}
W_{j}^{\mathrm{JBF}}(\mathbf{i})=h^{-1}(\mathbf{i}) \sum_{\mathbf{i}^{\prime} \in N_{\mathbf{i}}} W_{j}\left(\mathbf{i}^{\prime}\right) c\left(\mathbf{i}, \mathbf{i}^{\prime}\right) s\left(\mathbf{M}(\mathbf{i}), \mathbf{M}\left(\mathbf{i}^{\prime}\right)\right), \\
s\left(\mathbf{M}(\mathbf{i}), \mathbf{M}\left(\mathbf{i}^{\prime}\right)\right)=\exp \left(-\left(\mathbf{M}(\mathbf{i})-\mathbf{M}\left(\mathbf{i}^{\prime}\right)\right)^{2} / 2 \sigma_{\mathrm{R}}^{2}\right), \\
c\left(\mathbf{i}, \mathbf{i}^{\prime}\right)=\exp \left(-\left\|\mathbf{i}-\mathbf{i}^{\prime}\right\|_{2}^{2} / 2 \sigma_{\mathrm{D}}^{2}\right) \\
h(\mathbf{i})=\sum_{\mathbf{i}^{\prime} \in N_{\mathbf{i}}} c\left(\mathbf{i}, \mathbf{i}^{\prime}\right) s\left(\mathbf{M}(\mathbf{i}), \mathbf{M}\left(\mathbf{i}^{\prime}\right)\right) .
\end{aligned}
$$

The value of a filtered volume $W_{j}^{\mathrm{JBF}}(\mathbf{i})$ at voxel $\mathbf{i}$ is a weighted combination of values of the original volume $W_{j}$ belonging to a $3 \mathrm{D}$ neighborhood $N_{\mathbf{i}}$ around $\mathbf{i}$. The neighboring voxels are weighted with the MIP range similarity $s$ (with bandwidth $\sigma_{\mathrm{R}}$ ) and the spatial closeness $c$ (with bandwidth $\sigma_{\mathrm{D}}$ ) and normalized by dividing by the sum of all weights $h$. We denote one iteration of JBF by $\operatorname{JBF}\{\mathbf{w}\}$, which describes the application of Equation 16 to all sub-vectors $\mathbf{w}_{j}, j=1 \ldots N_{\mathrm{W}}$, followed by recomputing $\mathrm{M}$ according to Equation 15 for the next JBF iteration.

Figure 3 shows the MIPs of the initial FDK reconstruction of noisy data from a digital brain phantom before and after 3 iterations of JBF denoising. The JBF is similar to the time intensity profile similarity (TIPS) PCT noise reduction method by Mendrik et al. [19]. However, evaluating the TIPS measure between two voxels requires to calculate a sum of squared differences along the temporal dimension. The JBF has the same computational efficiency as standard 3D bilateral filtering and can also be implemented very similarly.

Elad [20] showed that the bilateral filter is related to Bayesian noise removal and derived the corresponding penalty function, which can apparently also be used to describe the JBF. We can combine the ML estimate of Equation 9 with a JBF penalty function $\mathcal{R}^{\mathrm{JBF}}(\mathbf{w})$ and enforce a physically correct solution by allowing only non-negative weights. This results in a maximum a-posteriori (MAP) estimation, which is a penalized weighted least squares (PWLS) problem. The constrained formulation of the PWLS problem is:

$$
\widetilde{\mathbf{w}}=\underset{\mathbf{w}}{\operatorname{argmin}} \mathcal{R}^{\mathrm{JBF}}(\mathbf{w}) \quad \text { s.t. } \mathcal{D}(\mathbf{w}) \leq \varepsilon \text { and } \mathbf{w} \geq 0 .
$$

It searches for the non-negative spline weights $\mathbf{w}$, which have the lowest JBF penalty at a data inconsistency $\mathcal{D}(\mathbf{w})$ of not more than $\varepsilon \in \mathcal{R}^{+}$. The data inconsistency parameter results from the sum of the minimally achievable inconsistency $\varepsilon_{\min }>0$ and an additional tolerance $\varepsilon_{\mathrm{t}} \geq 0: \varepsilon=\varepsilon_{\min }+\varepsilon_{\mathrm{t}}$. The minimal inconsistency $\varepsilon_{\min }$ will always be positive since noise, physical effects such as beam hardening, discretization, and the approximation of the TACs by the splines will not allow to find a solution with perfect data consistency in practical applications. The tolerance $\varepsilon_{\mathrm{t}}$ is a parameter to control smoothness and noise level in the brain tissue. For example, a higher $\varepsilon_{\mathrm{t}}$ achieves a solution with lower data consistency and lower JBF penalty, which means less noise but more blurring in the tissue. In this work, we do not solve Equation 17 directly. We rather search for a solution matching the goals of data consistency, edge-preserving smoothness, and positivity using the empirical approach discussed in subsection III-C.

\section{B. Basis Functions}

We evaluate the performance of different sets of basis functions to describe the TACs. We use basis functions with compact support, so that in the interpolation step (Equation 3 ) and in the update step (Equation 12) only the addends with non-zero basis weights have to be evaluated, which reduces the computational effort. Three different classes of basis functions are investigated in this work: asymmetric linear splines, symmetric linear splines, and cubic splines.

The first class are asymmetric linear functions from our previous work [9] shown in Figure 4a. The TACs are described by $N_{\mathrm{w}}=2 \cdot N_{\text {rot }}$ weight vectors $\mathbf{w}_{j}$. The knots of the basis 


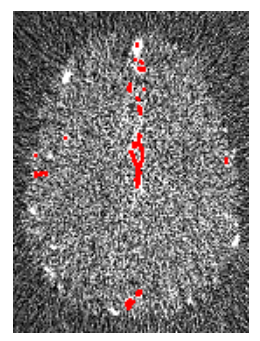

(a) noisy MIP

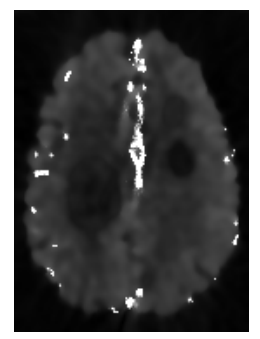

(b) denoised MIP

Figure 3: Maximum intensity projection (MIP) slices of a initial 4D perfusion volume with and without JBF noise reduction (window: [0 50] HU). The red pixels in the noisy MIP describe the segmented vessel structures.

functions are placed at the time points $\mathbf{t}^{\mathrm{w}}=\left[t_{1}^{\mathrm{w}} \cdots t_{N_{\mathrm{w}}}^{\mathrm{w}}\right]$, where:

$$
t_{j}^{\mathrm{w}}=\left\{\begin{array}{lll}
\frac{j-1}{2} \cdot\left(T_{\text {stop }}+T_{\text {rot }}\right)+0.25 \cdot T_{\text {rot }} & j \text { odd } \\
\left\lfloor\frac{j-1}{2}\right\rfloor \cdot\left(T_{\text {stop }}+T_{\text {rot }}\right)+0.75 \cdot T_{\text {rot }} & j \text { even. }
\end{array}\right.
$$

The TACs are described by linear interpolation between the knots which have nonuniform distance to each other. Therefore we have to define two different functions to describe the basis. If the knot index $j$ is odd then $b_{j}^{\mathrm{ALO}}(t)$ is used and if $j$ is even then $b_{j}^{\mathrm{ALE}}(t)$ is used. These basis functions are defined with $T_{1}=T_{\text {rot }} / 2, T_{2}=T_{\text {rot }} / 2+T_{\text {stop }}$, and $t^{\prime}=t-t_{j}^{\mathrm{W}}$ as:

$$
\begin{aligned}
& b_{j}^{\mathrm{ALO}}(t)= \begin{cases}1+t^{\prime} / T_{2} & -T_{2} \leq t^{\prime}<0, \\
1-t^{\prime} / T_{1} & 0 \leq t^{\prime} \leq T_{1}, \\
0 & \text { else }\end{cases} \\
& b_{j}^{\mathrm{ALE}}(t)= \begin{cases}1+t^{\prime} / T_{1} & -T_{1} \leq t^{\prime}<0, \\
1-t^{\prime} / T_{2} & 0 \leq t^{\prime} \leq T_{2}, \\
0 & \text { else. }\end{cases}
\end{aligned}
$$

Exceptions need to be defined for the beginning and for the end of the acquisition. For $j=1$, we set $T_{2}=T_{\text {rot }} / 4$ since we assume the TACs to rise from $0 \mathrm{HU}$ in the beginning. For $t>t_{N_{\mathrm{w}}}^{\mathrm{w}}$, we use a constant basis function. This reflects the assumption that we expect a steady-state plateau phase of residual contrast in the end. This type of basis functions avoids to place spline knots in the pauses between the acquisitions.

The second class of basis functions are linear spline functions with uniformly distributed knots placed in distance

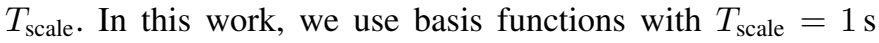
and $T_{\text {scale }}=2 \mathrm{~s}$, which are shown in Figure $4 \mathrm{~b}$. With the total scan time $T_{\text {scan }}$, the total number of splines used is $N_{\mathrm{w}}=\left\lfloor T_{\text {scan }} / T_{\text {scale }}\right\rfloor$ and the places of the spline knots are $t_{j}^{\mathrm{w}}=j \cdot T_{\text {scale }}, j=1 \ldots N_{\mathrm{w}}$. The linear basis functions are defined as:

$$
b_{j}^{\mathrm{LS}}(t)= \begin{cases}0 & t^{\prime} \geq 1 \\ 1-t^{\prime} & t^{\prime}<1\end{cases}
$$

with $t^{\prime}=\left|t-t_{j}^{\mathrm{w}}\right| / T_{\text {scale. Again we use a constant basis }}$ function for $t>t_{N_{\mathrm{w}}}^{\mathrm{W}}$.

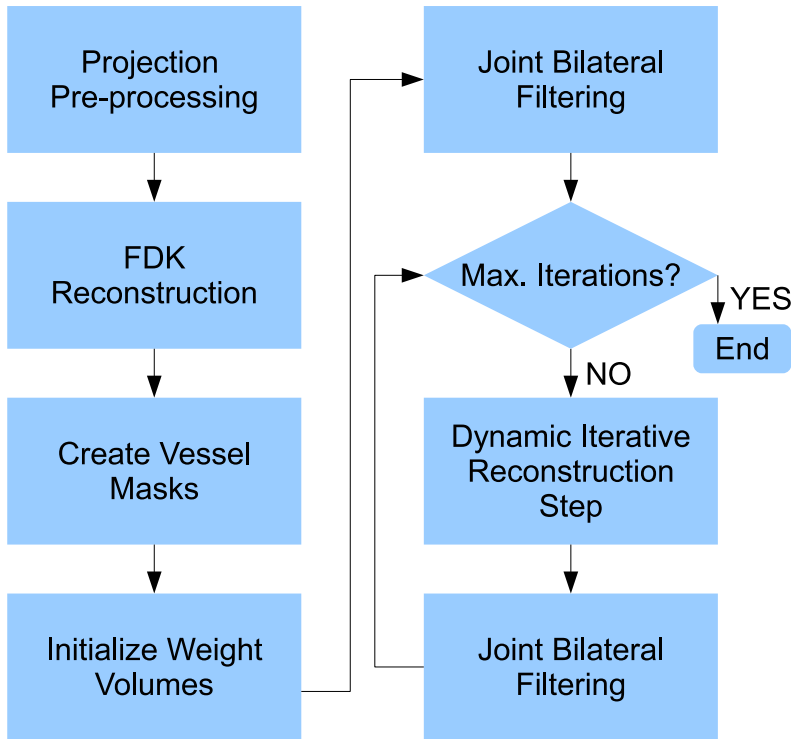

Figure 5: DIR-MAP algorithm flow chart.

The third class of basis functions are cubic spline functions with uniformly distributed knots, which are shown in Figure 4c. The knots are placed like the linear spline knots. The cubic spline basis functions are defined by the closed-form representation of the cubic B-spline [21]:

$$
b_{j}^{\mathrm{CS}}(t)= \begin{cases}0 & t^{\prime} \geq 2 \\ \left(2-t^{\prime}\right)^{3} / 6 & 2>t^{\prime} \geq 1, \\ \frac{2}{3}-t^{\prime 2}\left(1-\frac{1}{2} t^{\prime}\right) & t^{\prime}<1\end{cases}
$$

with $t^{\prime}=\left|t-t_{j}^{\mathrm{W}}\right| / T_{\text {scale }}$.

\section{Implementation Details}

This section describes the details of our implementation of the DIR-MAP algorithm. The algorithm finds an empirical approximation of the MAP estimate $\widetilde{\mathbf{w}}$ according to Equation 17 in a computational efficient way. A flow chart of the complete algorithm is shown in Figure 5 and a detailed overview of the single steps is shown in Figure 6. These steps are discussed below.

Initialization: In step 1 , the pure-contrast projection data $\mathbf{p}$ is computed from subtraction of the mask and bolus acquisitions. In case of real data, additional pre-processing is required, particularly to compensate for small motion of objects inside the field of view. These pre-processing steps are described in Appendix A. In step 2, all rotations are reconstructed using a standard short-scan FDK reconstruction method [16], [22]. The filtering step applies a Shepp-Logan filter kernel [23] multiplied with a Gaussian of variance $\sigma_{\mathrm{K}}^{2}$ controlling smoothness and noise level in the reconstructed volumes. For initialization, we use a sharp filter kernel with $\sigma_{\mathrm{K}}=0.25$ pixel to avoid blurring of the reconstructed volumes. In step 3, the temporal MIP $\mathrm{M}$ is computed and the binary vessel mask volume $\mathbf{v}^{\mathrm{V}}$ is created by thresholding $\mathrm{M}$ with the vessel threshold $\tau_{\mathrm{MIP}}$. To remove single voxels with MIP value above $\tau_{\text {MIP }}$ due to the heavy noise, $\mathbf{v}^{\mathrm{V}}$ is 


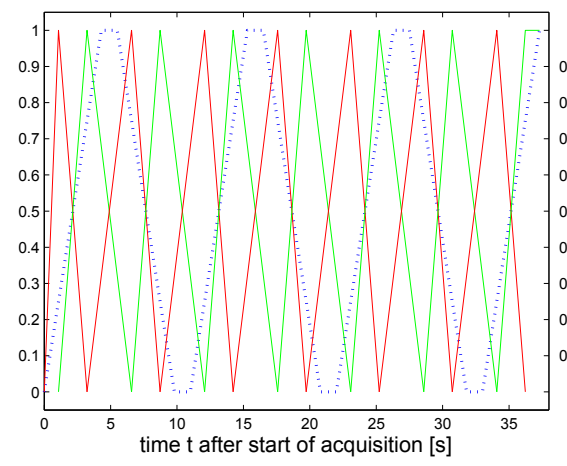

(a) Linear asymmetric splines

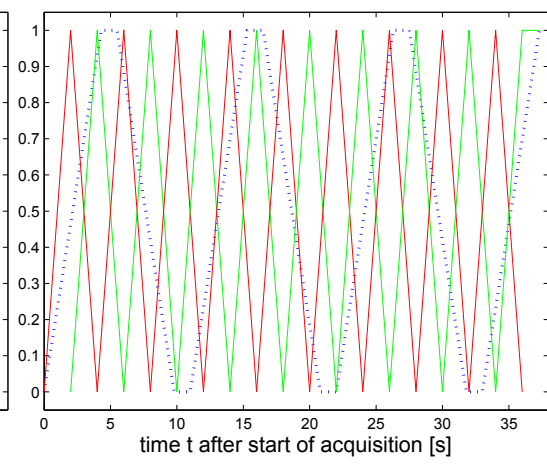

(b) Linear splines, $T_{\text {scale }}=2 \mathrm{~s}$

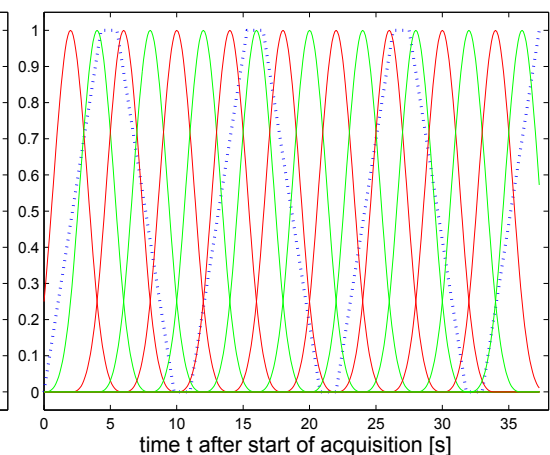

(c) Cubic splines, $T_{\text {scale }}=2 \mathrm{~s}$

Figure 4: Basis functions used to describe the reconstructed TACs. Red and green solid curves: basis functions. Blue dotted curves: relative angular $\mathrm{C}$-arm position.

processed by a 3D erosion and dilation operation. Figure 3a shows an example of a vessel mask thresholding. Accordingly, in step 4 vessel masks in projection space $\mathbf{v}_{i}^{\mathrm{P}}$ are computed for all $i=1 \ldots N_{\mathrm{P}}$ projections by a maximum intensity forward projection of $\mathbf{v}^{\mathrm{V}}$. Note that the acquisition geometry is the same for forward and each backward rotation, respectively. Thus practically only vessel masks for $2 \cdot N_{\text {proj }}$ projections have to be computed. In step 5, the weights $\mathrm{w}$ are initialized by the attenuation values from the initial FDK reconstructions. Therefore, initial TACs are interpolated from the FDK reconstructions. The spline weights $\mathbf{w}$ are matched by least squares fitting of the TACs described by $w$ to the initial TACs using singular value decomposition [24]. In step 6, the MIP $\mathrm{M}$ is denoised using bilateral filtering with range variance $\sigma_{\mathrm{R} 0}^{2}$ and domain variance $\sigma_{\mathrm{D}}^{2}$ and then the initial weights $\mathrm{w}$ are denoised using three iterations of JBF with range variance $\sigma_{\mathrm{R}}^{2}$, domain variance $\sigma_{\mathrm{D}}^{2}$, and guidance image $\mathrm{M}$ (step 7). The neighborhood $N_{\mathrm{i}}$ used for JBF is a 3D cube of $7 \times 7 \times 7$ voxels and the JBF was implemented GPU-based.

Dynamic Iterative Reconstruction: After the initialization steps, the algorithm iterates $N_{\text {it }}$ times (step 8). In each iteration, all rotations are processed subsequently (step 9). To improve convergence speed, the projections of one rotation are processed using an ordered subset (OS) [25] approach. The projections of each rotation are partitioned into $N_{\text {sub }}=10$ subsets $\mathcal{S}_{o, p}, o=1 \ldots N_{\text {rot }}, p=1 \ldots N_{\text {sub }}$, maximizing the difference of the projection acquisition angles in each subset. The subsets belonging to one rotation are processed subsequently (step 10). In step 11, temporary weight volumes $\mathbf{w}_{j}^{*}, j=1 \ldots N_{\mathrm{w}}$, are initialized to store the weight update values from one processed subset. Then, the algorithm iterates through the projections belonging to the current subset $\mathcal{S}_{o, p}$ (step 12). For each projection, the estimated attenuation values $\mathbf{x}$ for the projection acquisition time point $t_{q}^{\mathrm{P}}$ are computed by GPU-based interpolation (step 13). In the interpolation step, only the weight vectors associated with basis functions which are non-zero at $t_{q}^{\mathrm{P}}$ have to be considered. The interpolated volume is forward projected to compute the error image e, which is weighted with the statistical ray weights $D_{q}$ (step 14). The forward projection is computed using a ray-driven forward projector applying linear interpolation. In step 15,

\begin{tabular}{c|c||c|c} 
Parameter & Value & Parameter & Value \\
\hline \hline$N_{\text {it }}$ & 12 & $\tau_{\text {MIP }}$ & $55 \mathrm{HU}$ \\
\hline$\beta$ & $2.4 / N_{\text {proj }}$ & $\sigma_{\mathrm{R} 0}$ & 0.001 \\
\hline$\sigma_{\mathrm{D}}$ & $1.5 \mathrm{~mm}$ & $\sigma_{\mathrm{R}}$ & $1.25 \cdot 10^{-4}$
\end{tabular}

Table II: Parameters of the DIR-MAP algorithm.

vessel-masked back projection of the error image onto a temporary weight volume $\mathrm{w}^{\prime}$ is applied using a voxel-driven back projector. Note that in the practical implementation, only one back projection is required and the two operators $A_{q}^{T}$ are only shown to ease notation. Forward and back projection are implemented GPU-based as described in [26]. In step 16, the temporary weights are updated with the back projected error weights. Thereby the update vectors are weighted by the step size parameter $\beta$ and the basis function value $b_{j}\left(t_{q}^{P}\right)$. Thus, again only the weight vectors associated with non-zero basis functions have to be considered. After processing one subset, the weight vectors are updated from the temporary weight vectors in step 18 and non-negativity of all weights is enforced in step 19 to assure a physically correct solution. In step 22, JBF is applied to the weights after every three full iterations.

\section{Evaluation}

In the evaluation, we compare pure FDK reconstruction with FDK reconstruction followed by JBF (FDK-JBF) and the DIRMAP approach using different basis functions. The FDK-JBF method corresponds to the initialization part of the DIR-MAP approach (DIR-MAP with $N_{\text {it }}=0$ and linear asymmetric basis functions). Simulation data created from a realistic digital brain phantom and data from a clinical canine stroke model study is used.

\section{A. Digital Brain Perfusion Phantom}

1) Phantom Description: Classical digital CT phantoms, such as the Shepp-Logan phantom [23], usually consist of homogeneous structures. This highly favors reconstruction algorithms exploiting homogeneity like algorithms using compressed sensing based regularizers (e.g., total variation (TV) minimization [27]) and also algorithms using regularization by bilateral filtering. Thus, simple extensions to $4 \mathrm{D}$ dynamic 
1) Projection pre-processing to create contrast projection data $\mathbf{p}$

2) FDK reconstructions of each rotation: $\mathbf{x}_{i}^{*}=\operatorname{FDK}\left\{\mathbf{p}_{i}\right\}, i=1 \ldots N_{\text {rot }}$

3) Create MIP and volume vessel mask: $\mathbf{M}=\max \left\{\mathbf{x}_{i}^{*} \mid i=1 \ldots N_{\text {rot }}\right\}, \mathbf{v}^{\mathrm{V}}=\left(\mathbf{M}>\tau_{\mathrm{MIP}}\right)$, $\mathbf{v}^{\mathrm{V}} \in\{0,1\}^{\mathcal{S}_{\mathrm{V}}}$

4) Create projection vessel mask: $\mathbf{v}_{i}^{\mathrm{P}}=\left(A_{i} \mathbf{v}^{\mathrm{V}}>0\right), \mathbf{v}_{i}^{\mathrm{P}} \in\{0,1\}^{\mathcal{S}_{\mathrm{P}}}, i=1 \ldots N_{\mathrm{P}}$

5) Initialize weights $w$ from FDK reconstructions

6) Apply bilateral filter on $\mathrm{M}$ with $\sigma_{\mathrm{R} 0}$ and $\sigma_{\mathrm{D}}$

7) Apply 3 iterations of JBF on w with $\sigma_{\mathrm{R}}$ and $\sigma_{\mathrm{D}}$ : $\mathbf{w}=\operatorname{JBF}\{\mathrm{JBF}\{\mathrm{JBF}\{\mathbf{w}\}\}\}$

8) For $k=1 \ldots N_{\mathrm{it}}$

9) For $o=1 \ldots N_{\text {rot }}$

10) For $p=1 \ldots N_{\text {sub }}$

11) Initialize temporary weights: $\mathbf{w}_{j}^{*}=\mathbf{0}, \forall j=1 \ldots N_{\mathrm{w}}, \mathbf{w}_{j}^{*} \in \mathcal{R}^{\mathcal{S}_{\mathrm{v}}}$

12) For $q \in \mathcal{S}_{o, p}$

13) Interpolate dynamic volume at $t_{q}^{\mathrm{P}}$ : $\mathbf{x}=\sum_{j \in \mathcal{S}_{J}} b_{j}\left(t_{q}^{\mathrm{P}}\right) \mathbf{w}_{j}, \mathcal{S}_{J}=\left\{j \mid b_{j}\left(t_{q}^{\mathrm{P}}\right)>0\right\}$

14) Compute error image via forward projection: $\mathbf{e}=D_{q}\left(\mathbf{p}_{q}-A_{q} \mathbf{x}\right)$

15) Vessel-masked back projection of error image: $\mathbf{w}^{\prime}=\mathbf{v}^{\mathrm{V}} \otimes A_{q}^{T} \mathbf{e}+\neg \mathbf{v}^{\mathrm{V}} \otimes A_{q}^{T}\left(\neg \mathbf{v}_{q}^{\mathrm{P}} \otimes \mathbf{e}\right)$

16) Update temporary weight vectors: $\mathbf{w}_{j}^{*}=\mathbf{w}_{j}^{*}+\beta \cdot b_{j}\left(t_{q}^{P}\right) \cdot \mathbf{w}^{\prime}, \forall j \in \mathcal{S}_{J}$

17) End For

18) Update weight vectors:

$$
\mathbf{w}_{j}=\mathbf{w}_{j}+\mathbf{w}_{j}^{*}, \forall j=1 \ldots N_{\mathbf{w}}
$$

19) Assure non-negativity: $\mathbf{w}=\max \{\mathbf{w}, \mathbf{0}\}$

20) End For

21) End For

22) If $(k \bmod 3==0)$ apply JBF on w with $\sigma_{\mathrm{R}}$ and $\sigma_{\mathrm{D}}$ : $\mathbf{w}=\operatorname{JBF}\{\mathbf{w}\}$

23) End For

Figure 6: DIR-MAP algorithm. The symbol $\otimes$ describes element-wise multiplication, the symbol $\neg$ describes elementwise negation and max describes row-wise maximum selection.

phantoms do not allow for a meaningful evaluation since real clinical data do not exhibit such homogenous structures.

Thus, we use the realistic digital brain phantom from our previous work [28] (available online [29]), which has a complex structure similar to a real human brain and is an adopted version of the phantom by Riordan et al. [30]. It is based on segmented brain MRI scans from a healthy human volunteer using the Freesurfer software [31]. Inside the segmented brain, different tissue classes were annotated manually: healthy tissue, tissue with reduced $\mathrm{CBF}$, and tissue with severely reduced $\mathrm{CBF}$ and CBV. Different perfusion parameters were assigned to the annotated classes as shown in Table III. We created a $4 \mathrm{D}$ brain perfusion phantom by simulating TACs in tissue and vessels according to the annotated segmented brain. Compared to the phantom in [30], we further reduced the sparsity of the brain phantom using the MR data and varied the perfusion parameters according to the intervals shown in Table III. Details on the phantom creation, stroke annotation and sparsity reduction can be found in previous publications [30], [28].

We created dynamic projection data by forward projecting the $4 \mathrm{D}$ brain phantom according to the $\mathrm{C}$-arm acquisition protocol discussed in Section II. To simulate quantum noise, Poisson-distributed noise was added to the projection data. To simulate a realistic noise level, a real C-arm CT scan of a water cylinder phantom with the acquisition parameters shown in Table I was performed, but with a system dose of $0.36 \mu \mathrm{Gy} /$ projection. In the numerical studies the noise level of a lower system dose was simulated to investigate a potential dose reduction for future patient studies compared to the animal study in this work. The water cylinder projection data was reconstructed using the FDK algorithm and the standard deviation inside the homogenous water region was measured. Then projection data from a static version of the brain phantom containing only homogenous tissue structures was created. Poisson noise for a specific emitted photon density was added to the projection data and the data was reconstructed. The standard deviation inside the reconstructed homogenous brain phantom was measured and compared to the standard deviation of the real water cylinder phantom. The emitted photon density was then adapted until a similar standard deviation was measured. For a realistic noise level in the brain phantom, an emitted X-ray density of $2.1 \cdot 10^{5}$ photons per $\mathrm{mm}^{2}$ at the detector and a monochromatic photon energy of $60 \mathrm{keV}$ was assumed.

2) Investigations: We reconstructed the brain phantom projection data using the DIR-MAP approach with the linear asymmetric splines, linear splines with $T_{\text {scale }}=1 \mathrm{~s}$ and $T_{\text {scale }}=2 \mathrm{~s}$, as well as cubic splines with $T_{\text {scale }}=1 \mathrm{~s}$ and $T_{\text {scale }}=2 \mathrm{~s}$ as basis functions and also with the FDKJBF and the FDK algorithm. For DIR-MAP and FDK-JBF reconstruction, the parameters shown in Table II were used (with $N_{\text {it }}=0$ for FDK-JBF). The pure FDK reconstruction was done with a filter kernel with smoothness $\sigma_{\mathrm{K}}=1.25$ pixel. After reconstruction of the TACs, the perfusion parameters were calculated with our in-house perfusion analysis software as discussed in Appendix B. Reference perfusion maps were created with our software from the ground truth TACs.

For quantitative evaluation, the root mean square error (RMSE) over time between the reconstructed and the reference TACs was computed for the arterial input function (AIF) and the TACs of the brain tissue. Furthermore, we calculated the Pearson correlation (PC) and RMSE between the reconstructed and the reference perfusion maps (CBF, CBV, MTT, and TTP). We considered the perfusion map slices containing the annotated stroke-affected areas inside a sub-volume of size $256 \times 256 \times 18$. To calculate the PC and RMSE, we applied an automated region of interest (ROI) analysis with vascular pixel elimination (VPE) [3]. Therefore, air and vessel structures were excluded from the perfusion maps according to the brain segmentation. A rectangular grid with a line spacing of $8 \times 8 \mathrm{~mm}^{2}$ was used to subdivide each slice of the perfusion 


\begin{tabular}{c|c|c|c|c|c|c|} 
& \multicolumn{2}{|c|}{ Healthy } & \multicolumn{2}{c|}{ Reduced CBF } & Severely Reduced CBF/CBV \\
& WM & GM & WM & GM & WM & GM \\
\hline CBF $[\mathrm{ml} / 100 \mathrm{~g} / \mathrm{min}]$ & $25 \pm 14$ & $53 \pm 14$ & $7.5 \pm 4.25$ & $16 \pm 4.25$ & $2.5 \pm 1.4$ & $5.3 \pm 1.4$ \\
$\mathrm{CBV}[\mathrm{ml} / 100 \mathrm{~g}]$ & $1.9 \pm 0.9$ & $3.3 \pm 0.4$ & $1.7 \pm 0.9$ & $3 \pm 0.7$ & $0.42 \pm 0.2$ & $0.71 \pm 0.12$ \\
MTT $[\mathrm{s}]$ & $4.6 \pm 0.7$ & $3.7 \pm 0.7$ & $14 \pm 0.75$ & $11 \pm 0.75$ & $10 \pm 1$ & $8 \pm 1$
\end{tabular}

Table III: Perfusion parameters simulated in annotated tissue (WM = white matter, GM = gray matter). Right: example slice of the annotation (white: WM, gray: GM, purple: arteries, yellow: reduced $\mathrm{CBF}$, red: severely reduced $\mathrm{CBF} / \mathrm{CBV}$ ).
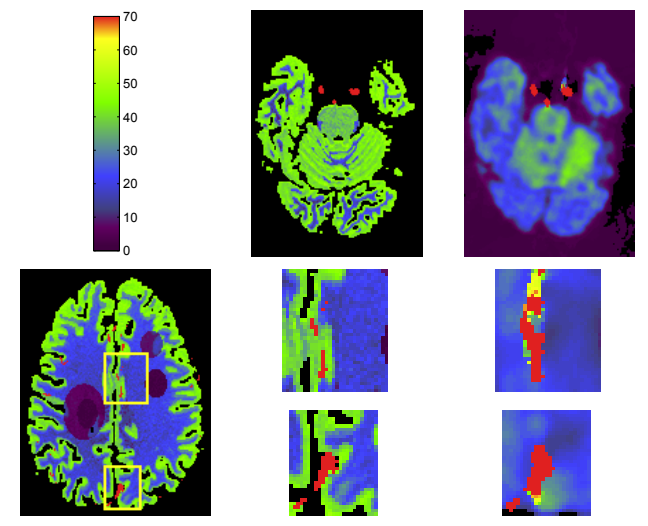

Reference

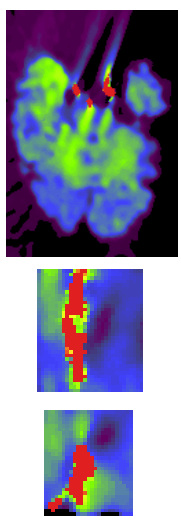

FDK-JBF

Figure 7: Artifacts in CBF maps (units: $\mathrm{ml} / 100 \mathrm{~g} / \mathrm{min}$ ) comparing DIR-MAP and FDK-JBF reconstructions.

map into square ROIs. For each ROI, the mean perfusion value was calculated for the reference and the reconstructed PCCT map. Then the PC and RMSE between the mean values of all valid ROIs in all slices were computed. ROIs including voxels not belonging to brain tissue according to the segmentation were ignored. The automated ROI analysis does not require manual selection of ROIs and is therefore userindependent. Furthermore, a qualitative comparison of artifacts around the arteries in DIR-MAP and FDK-JBF reconstructed CBF maps was done. These artifacts arise due to the high contrast dynamics in the arteries [32].

3) Results: Figure 8 shows the AIFs from DIR-MAP, FDK-JBF, and FDK reconstructions used to calculate the perfusion parameters compared to the reference curve. The quantitative results comparing the DIR-MAP, FDK-JBF, and FDK reconstructions are shown in Table IV. The CBF, CBV, MTT, and TTP perfusion maps from the three reconstruction algorithms compared to the reference maps are shown in Figure 9. Figure 7 compares artifacts around the arteries in CBF maps reconstructed with DIR-MAP and FDK-JBF.

\section{B. In Vivo Study}

1) Materials \& Methods: To validate the DIR-MAP algorithm under realistic conditions, we have conducted an in vivo brain perfusion study with canine ischemic stroke models, where we used PCT as reference for the validation. The ischemic stroke was induced in the healthy canines using the procedure discussed in [33]. Four hours after stroke creation, PCT was acquired and immediately followed by a PCCT.

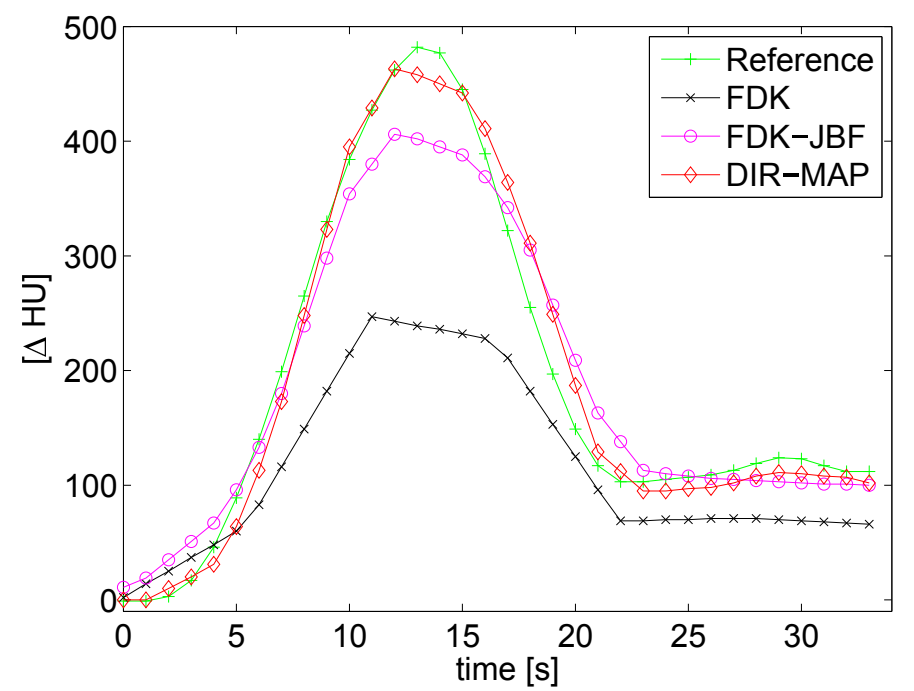

Figure 8: AIFs reconstructed from the digital brain phantom data with DIR-MAP (with linear asymmetric basis functions), FDK-JBF and FDK approach compared to the reference curve.

The contrast injection parameters were the same for both modalities and are summarized in Table V.

The PCCT data was acquired with a clinical C-arm angiography system (Artis zeego, Siemens AG, Forchheim, Germany) using the acquisition protocol described in Section II. The PCT was performed using a clinical 64-section volume CT scanner (GE Healthcare, Milwaukee, WI, USA) with continuous scanning for $50 \mathrm{~s}, 1 \mathrm{~s}$ per rotation, $80 \mathrm{kV}$ tube voltage and $200 \mathrm{~mA}$ tube current. The reconstructed data from the PCT exam covered 8 slices with a voxel size of $0.234 \times 0.234 \times 5 \mathrm{~mm}^{3}$ and was sampled in a temporal interval of $0.5 \mathrm{~s}$. The PCT data was denoised using the TIPS filter [19] with a spatial similarity kernel of bandwidth $\sigma_{\mathrm{D}}=1.5 \mathrm{~mm}$ and and a TIPS kernel of bandwidth $\sigma_{\text {TIPS }}=7.5 \mathrm{HU}$.

The PCCT data was pre-processed as described in Appendix $A$ and reconstructed using DIR-MAP and FDK-JBF with the parameters shown in Table II, but with $\tau_{\mathrm{MIP}}=155 \mathrm{HU}$ and $\sigma_{\mathrm{R}}=5 \cdot 10^{-4}$ (and $N_{\text {it }}=0$ for FDK-JBF). To calculate the perfusion maps from the reconstructed PCT and PCCT acquisitions, a TAC inside the basilar artery was selected as AIF and our in-house perfusion analysis software was used.

For quantitative evaluation, the PC and RMSE between the PCCT perfusion maps reconstructed with the DIR-MAP and the FDK-JBF approach and the PCT were computed. Therefore, the slice thickness of the PCCT volumes was aligned to the PCT slice thickness by applying a moving average 


\begin{tabular}{|c|c|c|c|c|c|c|c|}
\hline $\begin{array}{c}\text { Algorithm } \\
\text { Basis Functions }\end{array}$ & FDK & FDK-JBF & lin. asym. & lin. $T_{\text {scale }}=2 \mathrm{~s}$ & $\begin{array}{r}\text { DIR-MAF } \\
\text { lin. } T_{\text {scale }}=1 \mathrm{~s}\end{array}$ & cub. $T_{\text {scale }}=2 \mathrm{~s}$ & cub. $T_{\text {scale }}=1 \mathrm{~s}$ \\
\hline RMSE AIF $[\Delta \mathrm{HU}]$ & 135.1 & 49.9 & 26.7 & 26.5 & 33.6 & 27.0 & 31.1 \\
\hline RMSE Tissue [ $\Delta \mathrm{HU}]$ & 2.74 & 2.26 & 2.22 & 2.17 & 2.12 & 2.21 & 2.12 \\
\hline $\mathrm{PC} \mathrm{CBF}$ & 0.85 & 0.87 & 0.92 & 0.91 & 0.89 & 0.91 & 0.90 \\
\hline RMSE CBF $[\mathrm{ml} / 100 \mathrm{~g} / \mathrm{min}]$ & 8.4 & 4.6 & 3.7 & 3.9 & 4.4 & 3.7 & 4.0 \\
\hline PC CBV & 0.83 & 0.86 & 0.88 & 0.88 & 0.86 & 0.87 & 0.88 \\
\hline RMSE CBV [ml/100 g] & 0.80 & 0.62 & 0.47 & 0.44 & 0.45 & 0.46 & 0.43 \\
\hline RMSE MTT [s] & 1.13 & 1.18 & 1.03 & 0.92 & 0.90 & 0.97 & 0.86 \\
\hline PC TTP & 0.75 & 0.80 & 0.84 & 0.84 & 0.80 & 0.86 & 0.79 \\
\hline RMSE TTP [s] & 1.11 & 0.96 & 0.78 & 0.78 & 0.77 & 0.78 & 0.80 \\
\hline
\end{tabular}

Table IV: Quantitative results digital brain phantom (PC: Pearson correlation, RMSE: root mean square error).

\begin{tabular}{c|c} 
contrast medium & $370 \mathrm{mgI} / \mathrm{ml}$ \\
\hline injection type & intravenous \\
\hline injection rate & $2.0 \mathrm{ml} / \mathrm{s}$ \\
\hline total contrast volume & $16 \mathrm{ml}$ \\
\hline total saline chase volume & $10 \mathrm{ml}$ \\
\hline X-ray delay & $5 \mathrm{~s}$
\end{tabular}

Table V: Canine study injection protocol.

filter (kernel size $5 \mathrm{~mm}$ ) perpendicular to the orientation of the reconstructed slices. Then, the PCCT volumes were registered to the PCT volumes using a rigid 3D-3D registration. The registration matrix was computed between mask volumes from the PCCT and PCT acquisition. The PC and RMSE were again computed using the automated ROI analysis described in Section IV-A2. Since there is no ground truth segmentation for the canine brains, the air and bone structures were found by thresholding a mask volume. Then remaining tissue not belonging to the brain was removed manually. The vascular structures were removed by excluding voxels with $\mathrm{CBV}$ values higher than $8 \mathrm{ml} / 100 \mathrm{~g}$ in the CBV PCT map. The ROI size was set to $2 \times 2 \mathrm{~mm}^{2}$.

2) Results: Figure 10 shows examples of an AIF from the PCCT acquisition of a canine reconstructed with DIR-MAP and FDK-JBF and from the PCT acquisition for comparison. In Figure 11, co-registered perfusion maps from PCCT acquisition reconstructed with DIR-MAP and FDK-JBF and from PCT acquisition are shown. The quantitative results measuring the PC and RMSE between the maps are given in Table VI. Figure 12 illustrates 3D perfusion maps reconstructed with DIR-MAP in different viewing directions.

The reconstructions were performed on a laptop computer with an Intel i7 M $6202 \times 2.72 \mathrm{GHz}$ CPU, 8 GB RAM, and an Nvidia Quadro FX 880M graphics chip set. The reconstruction of a typical 4D volume of size $256 \times 256 \times 150$ voxels with linear asymmetric basis functions took about $36 \mathrm{~min}$ using a non-optimized DIR-MAP implementation and about 4.5 min using the FDK-JBF approach, where the projection pre-processing and perfusion parameter computations are not included.

\section{DISCUSSION}

The results of the digital brain phantom study show the potential of the dynamic DIR-MAP algorithm to improve PCCT compared to pure FDK reconstruction and also to FDKJBF reconstruction. Comparing the AIFs shown in Figure 8,

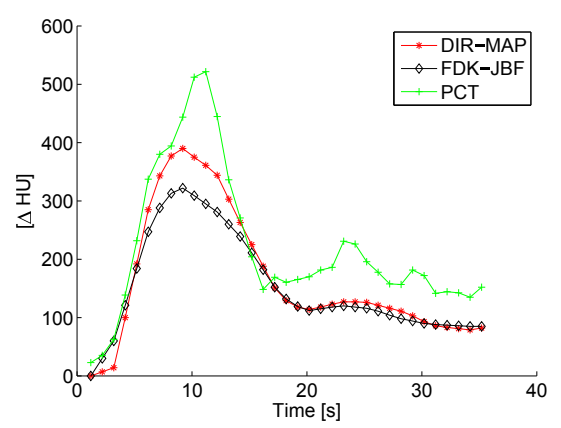

Figure 10: Examples of an arterial input function obtained from a canine (canine $\mathrm{C}$ in Table VI) reconstructed with DIRMAP and FDK-JBF. Also, the curve from the perfusion CT (PCT) is shown.

the AIF reconstructed with the DIR-MAP approach is the best approximation of the reference AIF with the lowest underestimation of the peak. Pure FDK reconstruction with a smooth filter kernel $\left(\sigma_{\mathrm{K}}=1.25\right)$ results in a severe underestimation of the AIF. The underestimation caused by the smooth filter kernel can be avoided by using a sharp reconstruction kernel $\left(\sigma_{\mathrm{K}}=0.25\right)$ followed by denoising with JBF. However, the dynamic DIR-MAP approach can still perceptibly improve the AIF estimation. Also, PC and the RMSE of all reconstructed maps are the best for DIR-MAP (Table IV), if linear asymmetric basis functions are used; e.g. the CBF PC increases from 0.85 (FDK) to 0.87 (FDK-JBF) and to 0.92 (DIR-MAP), and the RMSE error decreases from 8.4 to 4.6 to $3.7 \mathrm{ml} / 100$ $\mathrm{g} / \mathrm{min}$, respectively. Furthermore, the results in Table IV also show that the simple linear asymmetric basis functions are able to provide sufficiently accurate perfusion maps, and more complex basis functions such as cubic splines do not improve them perceptibly. The perfusion maps reconstructed with DIRMAP and FDK-JBF compared to the FDK maps (Figure 9) are smoother, the stroke-affected areas are better separated from the healthy tissue and the vascular structures, which are visible as the red structures in the CBF and CBV maps, are not blurred into the brain tissue. Comparing the DIR-MAP with the FDK-JBF maps, the DIR-MAP approach avoids the artifacts around the arteries, which appear in filtered back projection algorithms like FDK around structures with high contrast dynamics (for a detailed analysis of such artifact see [32]). Figure 7 shows these artifacts in more details. Also, there is less overestimation of the perfusion parameters in the 


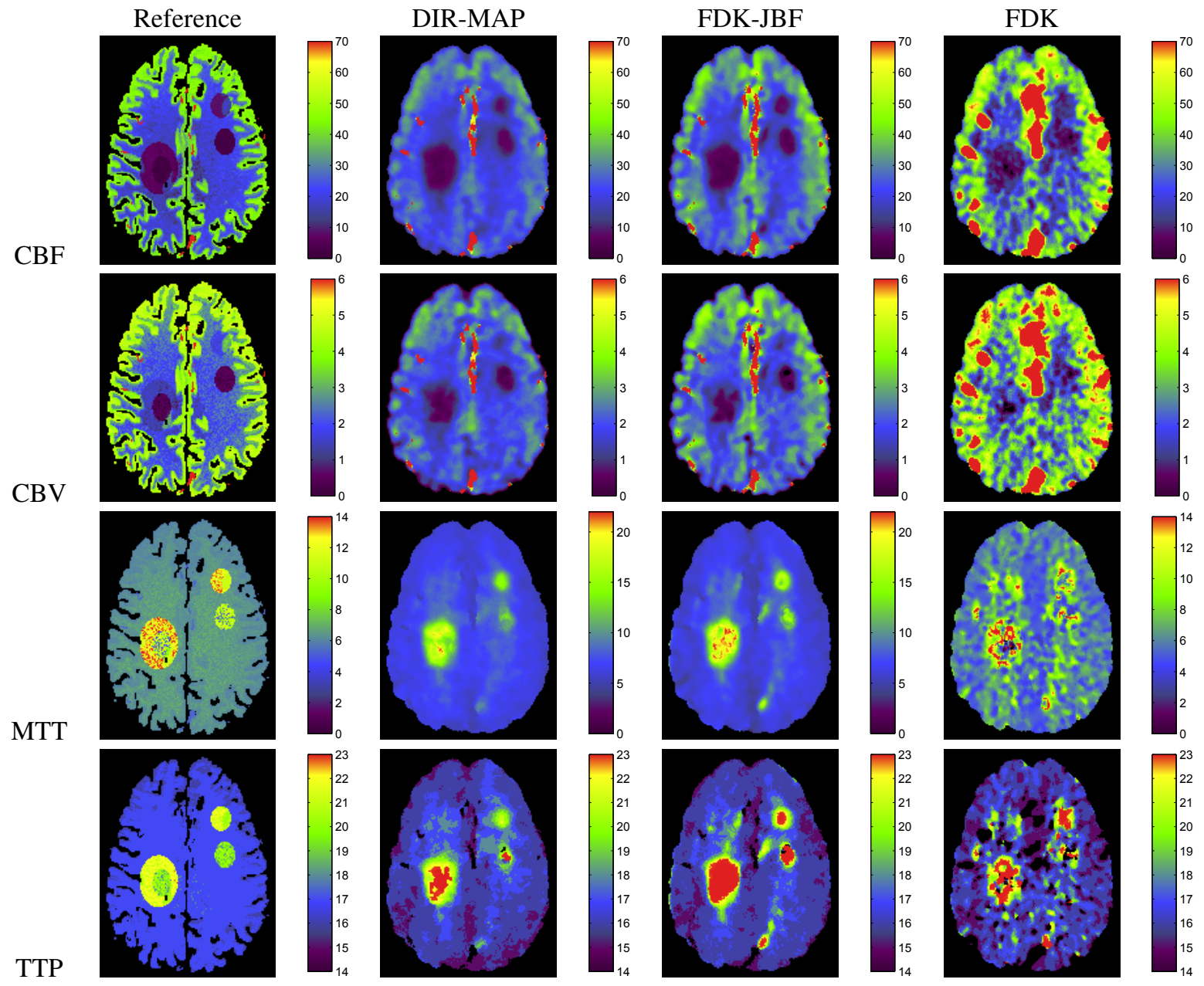

Figure 9: CBF (units: $\mathrm{ml} / 100 \mathrm{~g} / \mathrm{min}$ ), CBV (units: $\mathrm{ml} / 100 \mathrm{~g}$ ), MTT (units: s), and TTP (units: s) perfusion maps from digital brain perfusion phantom data reconstructed with DIR-MAP (with linear asymmetric basis functions), FDK-JBF, and FDK algorithm.

\begin{tabular}{ccc|cccccc|c} 
Perfusion Parameter & \multirow{2}{*}{ Reconstruction Algorithm } & & $\mathrm{A}$ & $\mathrm{B}$ & $\mathrm{C}$ & $\mathrm{D}$ & $\mathrm{E}$ & $\mathrm{F}$ & Mean $\pm \mathrm{SD}$ \\
\hline \hline \multirow{3}{*}{ CBF } & \multirow{2}{*}{ DIR-MAP } & PC & 0.80 & 0.63 & 0.71 & 0.82 & 0.75 & 0.66 & $0.73 \pm 0.08$ \\
& & RMSE & 10.6 & 9.9 & 11.4 & 9.2 & 12.4 & 8.2 & $10.3 \pm 1.5$ \\
\cline { 2 - 9 } & \multirow{2}{*}{ FDK-JBF } & PC & 0.80 & 0.60 & 0.63 & 0.77 & 0.71 & 0.63 & $0.69 \pm 0.08$ \\
& & RMSE & 10.5 & 10.1 & 18.3 & 14.9 & 14.0 & 8.4 & $12.7 \pm 3.7$ \\
\cline { 3 - 9 } CBV & \multirow{2}{*}{ DIR-MAP } & PC & 0.72 & 0.50 & 0.61 & 0.79 & 0.61 & 0.61 & $0.64 \pm 0.10$ \\
& & RMSE & 0.92 & 0.95 & 1.30 & 1.30 & 1.84 & 1.22 & $1.25 \pm 0.33$ \\
\cline { 2 - 9 } & \multirow{2}{*}{ FDK-JBF } & PC & 0.71 & 0.54 & 0.58 & 0.79 & 0.64 & 0.64 & $0.65 \pm 0.09$ \\
& & RMSE & 1.88 & 1.53 & 1.78 & 1.77 & 2.07 & 1.43 & $1.74 \pm 0.23$ \\
\hline \multirow{3}{*}{ MTT } & \multirow{2}{*}{ DIR-MAP } & PC & 0.61 & 0.60 & 0.74 & 0.56 & 0.70 & 0.49 & $0.62 \pm 0.09$ \\
& & RMSE & 4.77 & 3.25 & 3.15 & 3.79 & 3.40 & 2.21 & $3.43 \pm 0.84$ \\
\hline \multirow{3}{*}{ FDK-JBF } & PC & 0.14 & 0.27 & 0.12 & 0.41 & 0.60 & 0.42 & $0.33 \pm 0.18$ \\
& & RMSE & 11.02 & 5.44 & 8.00 & 5.44 & 4.00 & 3.08 & $6.16 \pm 2.90$ \\
\hline \multirow{2}{*}{ TTP } & \multirow{2}{*}{ DIR-MAP } & PC & 0.67 & 0.62 & 0.75 & 0.56 & 0.77 & 0.17 & $0.59 \pm 0.22$ \\
& & RMSE & 5.79 & 3.75 & 4.15 & 5.61 & 4.08 & 2.74 & $4.35 \pm 0.97$ \\
\cline { 2 - 8 } & \multirow{2}{*}{ FDK-JBF } & PC & 0.59 & 0.59 & 0.58 & 0.51 & 0.71 & 0.24 & $0.54 \pm 0.16$ \\
& & RMSE & 5.86 & 4.64 & 5.26 & 5.96 & 4.46 & 3.39 & $4.39 \pm 1.16$
\end{tabular}

Table VI: PC and RMSE between perfusion parameters measured with CT and C-arm CT in six canines with induced stroke (labeled from A to F) reconstructed with DIR-MAP and FDK-JBF (SD: standard deviation). The total number of ROI samples used to measure PC and RMSE is $n=13381$. 


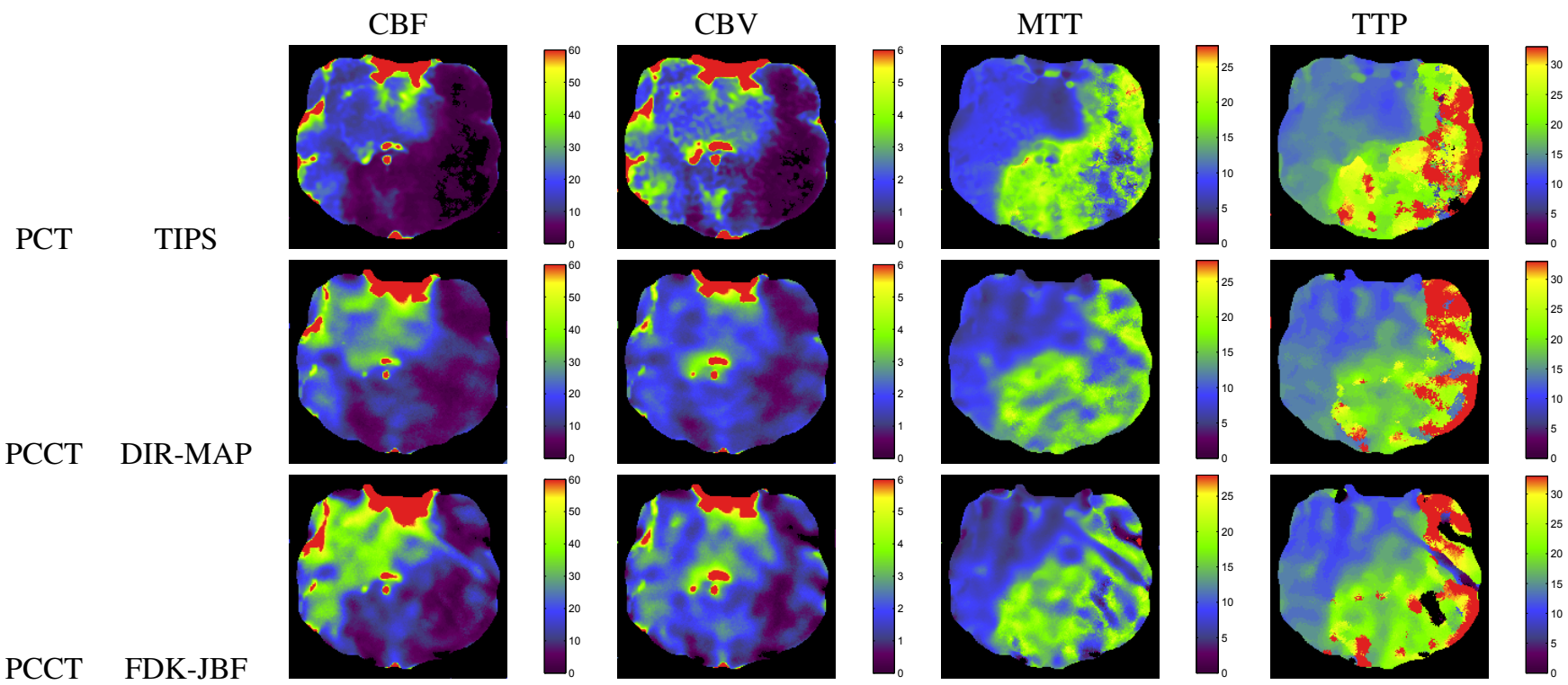

Figure 11: CBF (units: $\mathrm{ml} / 100 \mathrm{~g} / \mathrm{min}$ ), CBV (units: $\mathrm{ml} / 100 \mathrm{~g}$ ), MTT (units: s), and TTP (units: s) maps of canine C with an induced ischemic stroke obtained with perfusion CT and perfusion C-arm CT (PCCT). The PCCT maps were reconstructed with the DIR-MAP and the FDK-JBF technique and registered to the PCT maps.
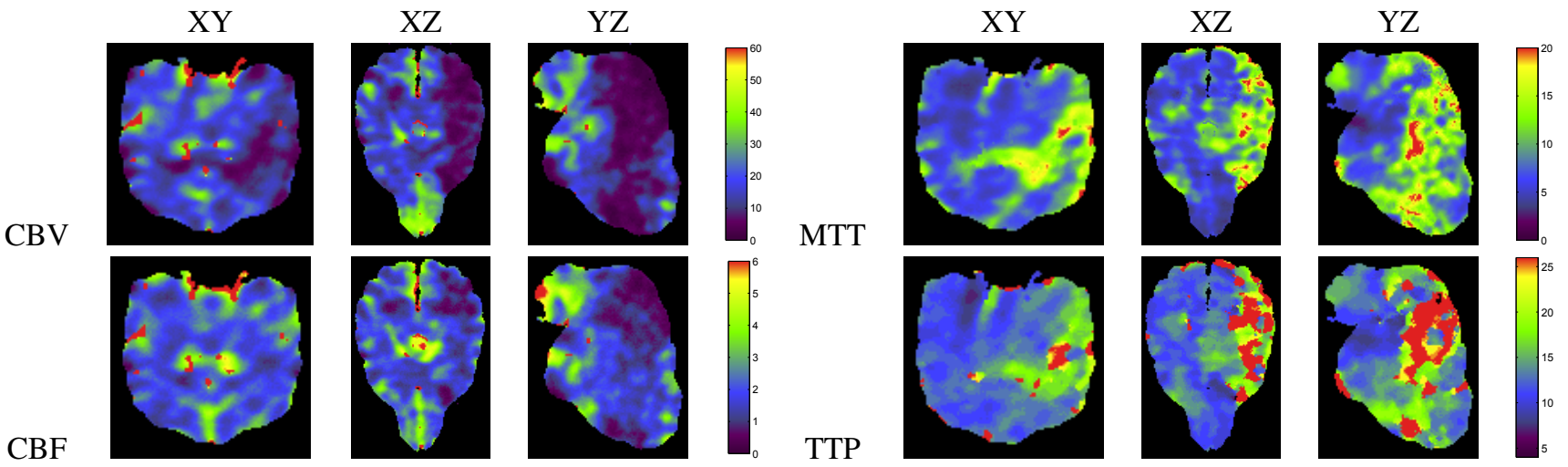

Figure 12: CBV (units: $\mathrm{ml} / 100 \mathrm{~g}$ ), CBF (units: $\mathrm{ml} / 100 \mathrm{~g} / \mathrm{min}$ ), MTT (units: s), and TTP (units: s) maps in XY, XZ and YZ viewing directions of canine $\mathrm{C}$ obtained with perfusion $\mathrm{C}$-arm $\mathrm{CT}$. The maps were reconstructed with the DIR-MAP technique and an isotropic voxel size of $0.5^{3} \mathrm{~mm}^{3}$.

DIR-MAP CBF and CBV maps, which corresponds to the improved RMSE shown in Table IV. The improved RMSE can be attributed to the higher peak in the AIF estimation compared to the FDK-JBF result, since the algorithm used to calculate the perfusion parameters uses the AIF to normalize the perfusion data (see Appendix B for details). In addition, the DIR-MAP maps make an even less noisier impression than the FDK-JBF maps.

The results of the canine study confirm the results of the simulation study that the dynamic DIR-MAP approach can improve PCCT compared to the static FDK-JBF technique. The PC and RMSE between the PCT and PCCT acquisitions are improved for all perfusion parameters, except the $\mathrm{PC}$ for $\mathrm{CBV}$. However, CBV is not a dynamic perfusion parameter and can also be measured using steady-state acquisitions [34]. Thus, we do not necessarily expect improvements from a dynamic approach. The higher peak in the DIR-MAP AIF estimation (see Figure 10) improves the RMSE of the CBF and CBV maps. The canine perfusion maps presented in Figure 11 also confirm the simulation results. The streaking artifacts arising from the big vessels in the upper middle of the maps are reduced considerably by the DIR-MAP approach. Furthermore, the CBF and CBV values are less overestimated in DIR-MAP than in FDK-JBF PCCT maps when comparing to the PCT maps.

Figure 12 shows the 3D perfusion maps of a PCCT acquisition reconstructed with DIR-MAP from different viewing directions. This highlights another advantage of PCCT compared to PCT: the possibility to reconstruct 3D perfusion maps with high resolution in $\mathrm{Z}$ (axial) direction. Since this is not possible with PCT, no reference maps can be provided. However, the extend of the stroke in the CBF, MTT, and TTP maps looks very similar. These parameters are computed in a very different way from the reconstructed TACs: CBF and 
MTT are computed using a deconvolution-based approach and TTP simply corresponds to the temporal position of the peak of the tissue TAC (see Appendix B). Therefore, we can consider the similarity of both maps as an indication for the correctness of our PCCT acquisition and reconstruction method.

In [3], first in vivo results of a PCCT study with healthy pigs were presented. A novel approach for PCCT was used, which combines interleaved scanning with partial volume reconstruction. However, the interleaved scanning approach increases the X-ray and contrast agent dose. Furthermore, the technique is based on the assumption that the contrast TACs are reproducible in subsequent PCCT acquisitions, which is not assured in clinical practice, since for example blood circulation parameters may change. In this work, we present an algorithmic dynamic reconstruction approach with the potential to compensate the low $\mathrm{C}$-arm rotation speed without repeated scanning.

The DIR-MAP algorithm is an empirical technique, which alternately maximizes data consistency and minimizes the Bayesian regularization term by bilateral filtering. It is not known if and how our approach converges and finds an solution of Equation 17 at a certain data inconsistency $\varepsilon$. For static reconstruction with TV regularization, algorithms have been proposed seeking for a minimal TV solution at certain $\varepsilon$ by adapting the TV step size to the data consistency [27], [35]. For bilateral filtering, such algorithms are not known to the authors. We use bilateral filtering as it is a very intuitive tool and the effect of its parameters on the filtered image are predictable. Furthermore, it provides an apparent possibility to use a guidance image. Our evaluation shows the potential of our empirical approach to enhance the reconstructed perfusion maps. An algorithm directly seeking an optimal solution of Equation 17 might help to further improve the results. However, even when directly solving Equation 17, the selection the appropriate data inconsistency tolerance $\varepsilon_{\mathrm{t}}$ is still an empirical problem.

\section{SUMmary \& CONCLUSIONS}

Brain perfusion measurement using C-arm CT (PCCT) is a promising new technique which allows the measurement of the dynamic perfusion values during interventional procedures with full brain coverage and in high resolution in axial direction. However, reconstruction of reliable perfusion maps from $\mathrm{C}$-arm acquisitions is a challenging task due to the low rotation speed of the $\mathrm{C}$-arm system and the low contrast level of the time attenuation curves (TACs) in the brain tissue.

In this work, we introduced a novel iterative reconstruction algorithm for PCCT to overcome these limitations. The algorithm is based on the iterative parameter estimation technique described by Neukirchen et al. [6]. In contrast to [6], we developed the algorithm for a real 3D C-arm cone beam geometry instead of a 2D parallel beam geometry and used an acquisition protocol available in state-of-the art clinical C-arm systems, including pauses between the acquisitions. We used spline basis functions with compact support to describe the TACs, which reduces the computational effort compared to the non-compact basis functions used in [6]. The back projection step was modified to avoid streaking artifacts around high contrast vessel structures in the cone beam reconstructions. To increase the contrast-to-noise ratio (CNR) in the tissue TACs, we applied a non-linear regularization based on joint bilateral filtering (JBF) [10] and statistical ray weighting. To handle the high computational effort of dynamic iterative reconstruction, the most computationally expensive steps were implemented using the Nvidia CUDA programming language.

The novel algorithm was evaluated extensively with a realistic digital brain phantom similar as described by Riordan et. al [30] and data from an in vivo study with canine stroke models. Both evaluations showed that the DIR-MAP algorithm consistently outperforms static FDK reconstruction, even if the FDK data is also processed with the JBF denoising. The results from the animal study are particularly promising: we received similar average Pearson correlation of the PCCT maps and the reference PCT maps in the canine study as Fieselmann et al. [3] in a pig study. However, in [3] an interleaved scanning approach with the need for repeated contrast injections and scanning sequences is applied. The average PC for CBF is 0.73 , which is achieved in [3] with three interleaved scans, and 0.64 for CBV, which is achieved in [3] with two interleaved scans. This comes at the price of an increased computational complexity of the iterative reconstruction method. Thus, further code optimization and the use of high end hardware will be required for interventional use. Future development of $\mathrm{C}$-arm hardware, such as faster rotations or avoiding the pauses between the rotations, will help to improve the PCCT measurement. To show the clinical applicability of our PCCT acquisition and reconstruction method, validation with human patient studies is necessary and will be carried out in the future.

\section{ACKNOWLEDGMENTS}

We would like to thank Dr. Charles Strother and his research team at the University of Wisconsin for contributing the experimental canine ischemic perfusion study data used for this project and André Aichert for helpful advice during preparation of the manuscript.

\section{APPENDIX}

\section{A. Projection Pre-Processing for In-Vivo Data}

To reconstruct in-vivo data, the projections acquired with the C-arm system need to be pre-processed. The usual preprocessing steps for $\mathrm{C}$-arm projection data, including logarithmic processing, overexposure, scatter, and beam hardening correction [36], are applied to compute the attenuation line integrals. To compensate for small movements of the animal (or objects in the acquired scene) between the mask and the contrast-enhanced acquisitions, a non-rigid 2D-2D motion correction between the static and the contrast-enhanced projections is applied [37] before the projection subtraction. However, this correction is not exact, since correction of 3D motion in the 2D projection space is ambiguous.

To avoid artifacts in the reconstructed volumes due to false attenuation values in the subtracted data caused by motion, we apply a correction step for invalid pixels in the subtracted 
projections. First, a volume vessel mask $\mathbf{v}^{\mathrm{V}}$ similar to step 2 of the DIR algorithm (Figure 6) is created. Then, all rotations $N_{\text {rot }}$ are processed subsequently as follows: first, the volume $\hat{\mathbf{x}}$ is reconstructed from the subtracted projections of the rotation using the FDK algorithm. In the next step, all voxels in the reconstructed volume $\hat{\mathbf{x}}$ which do not belong to a vessel structure (as indicated by $\mathbf{v}^{\mathrm{V}}$ ) are set to zero, such that only the vessels remain and the tissue attenuation and especially the artifacts are suppressed. Afterwards all projections $\mathbf{p}_{i}$ belonging to the rotation are corrected by detecting and replacing invalid pixel values. To detect invalid values, the vessel projection $\hat{\mathbf{p}}_{i}$ is created by forward projecting $\hat{\mathbf{x}}$. Let $p_{i, k}=\mathbf{p}_{i}(k), k=1 \ldots \mathcal{S}_{\mathrm{P}}$, denote the value of pixel $k$ in projection $i$ and $\hat{p}_{i, k}=\hat{\mathbf{p}}_{i}(k)$ the corresponding value in the vessel projection. If $\hat{p}_{i, k}=0$, the pixel does not belong to a vessel and is compared to the thresholds $\tau_{1}^{\mathrm{C}} \in \mathcal{R}^{-}$and $\tau_{\mathrm{h}}^{\mathrm{C}} \in \mathcal{R}^{+}$defining reasonable upper and lower bounds of values for line integrals not intersecting with high contrast vessels. If $p_{i, k}<\tau_{1}^{\mathrm{C}}$ or $p_{i, k}>\tau_{\mathrm{h}}^{\mathrm{C}}$, then $p_{i, k}$ is marked as an invalid pixel. If $\tau_{1}^{\mathrm{C}}<p_{i, k}<0$, we assume that the pixel value is invalid not because of motion, but only due to noise and $p_{i, k}$ is set to zero. If $\hat{p}_{i, k}>0$, the pixel belongs to a vessel and is marked as invalid if $\left|p_{i, k}-\hat{p}_{i, k}\right|>\tau_{\mathrm{v}}^{\mathrm{C}}$ or $p_{i, k}<0$. The threshold $\tau_{\mathrm{v}}^{\mathrm{C}}$ defines a reasonable range of variation in contrast attenuation for pixels belonging to rays intersecting with vessels. The variation stems from the changing attenuation of the vessels during the acquisition and the tissue attenuation, which are both not considered in the static volume $\hat{\mathbf{x}}$. After the invalid pixels on the projection are marked, they are replaced by row-wise 1-D linear interpolation between the closest valid pixels. In this work, we used the following thresholds: $\tau_{1}^{\mathrm{C}}=-0.08, \tau_{\mathrm{h}}^{\mathrm{C}}=0.8$, and $\tau_{\mathrm{v}}^{\mathrm{C}}=0.15$.

Figure 13 shows an example of how the invalid pixel correction can help to improve the reconstructed maps. Due to motion of a plastic tube between the mask and contrast-filled projections, there are invalid pixels with too high and too low attenuation values in some of the subtracted projections. This leads to streak artifacts in the resulting CBF map. Using the invalid pixel correction, the streak artifacts can be avoided.

\section{B. Perfusion Parameter Calculation}

To compute the perfusion parameters, the reconstructed TACs were sampled with a temporal resolution of $1 \mathrm{~s}$ from the weighted spline description. For the FDK algorithm, the sampling was done using linear interpolation between the reconstructed rotations, where each reconstructed rotation represents TAC samples at the mid time point of its acquisition. Our in-house perfusion analysis software calculated the CBF, CBV, and MTT maps using the truncated SVD algorithm [38] based on the indicator-dilution theory [39]. The TTP maps were computed by determining the time from the mid time point of the first acquisition to the peak of the reconstructed TAC. For robust peak detection in noisy TACs, a cubic Savitzky Golay filter [40] of size 25 samples was applied prior to the peak search.

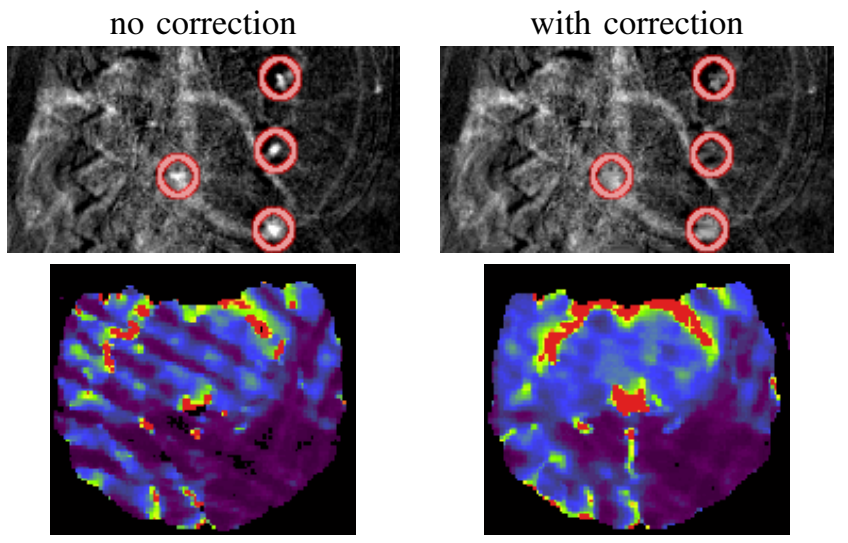

Figure 13: Invalid pixel correction. Upper left image: subtracted projection image with invalid pixel values (marked with red circles), which are too high due to a plastic tube which has moved between the mask and the contrast-filled projections. Upper right image: corrected subtracted projection image. Lower left image: CBF map reconstructed from uncorrected projections. Lower right image: $\mathrm{CBF}$ map reconstructed from corrected projections.

\section{REFERENCES}

[1] R. G. González, J. A. Hirsch, W. J. Koroshetz, M. H. Lev, and P. W. Schaefer, Eds., Acute Ischemic Stroke, 1st ed. Berlin, Germany: Springer, 2006.

[2] A. Furlan, R. Higashida, L. Wechsler, M. Gent, H. Rowley, C. Kase, M. Pessin, A. Ahuja, F. Callahan, W. M. Clark, F. Silver, and F. Rivera, "Intra-arterial prourokinase for acute ischemic stroke: The PROACT II study: A randomized controlled trial," JAMA, vol. 282, pp. 2003-2011, 1999.

[3] A. Fieselmann, A. Ganguly, Y. Deuerling-Zheng, M. Zellerhoff, C. Rohkohl, J. Boese, J. Hornegger, and R. Fahrig, "Interventional 4D C-arm CT perfusion imaging using interleaved scanning and partial reconstruction interpolation," IEEE Trans Med Imaging, vol. 31, no. 4, pp. $892-906,2012$.

[4] A. Ganguly, A. Fieselmann, M. Marks, J. Rosenberg, J. Boese, Y. Deuerling-Zheng, M. Straka, G. Zaharchuk, R. Bammer, and R. Fahrig, "Cerebral CT perfusion using an interventional C-arm imaging system: Cerebral blood flow measurements," Am J Neuroradiol, vol. 35, pp. 1525-1531, 2011.

[5] S. Serowy, O. Gurvit, M. Skalej, and G. Rose, "Jacobi-like solution to the model based tomographic x-ray perfusion imaging," in IEEE NSS MIC Conference Record, 2007, pp. 3085-3088.

[6] C. Neukirchen, M. Giordano, and S. Wiesner, "An iterative method for tomographic X-ray perfusion estimation in a decomposition model-based approach," Medical Physics, vol. 37, no. 12, pp. 6125-6141, 2010.

[7] A. Kak and M. Slaney, Principles of computerized tomographic imaging. Piscataway, NJ: IEEE Service Center, 1988.

[8] A. Fieselmann and M. T. Manhart, "Interventional perfusion imaging using C-Arm CT," Current Medical Imaging Reviews, vol. -, pp. -, 2013, (in press)

[9] M. T. Manhart, M. Kowarschik, A. Fieselmann, Y. Deuerling-Zheng, and J. Hornegger, "Fast dynamic reconstruction algorithm with joint bilateral filtering for perfusion C-arm CT," in IEEE NSS MIC Conference Record, Anaheim, USA, 2012, pp. 2304-2311.

[10] G. Petschnigg, M. Agrawala, H. Hoppe, R. Szeliski, M. Cohen, and $\mathrm{K}$. Toyama, "Digital photography with flash and no-flash image pairs," in Proc. ACM SIGGRAPH, vol. 23, no. 3, 2004, pp. 664-672.

[11] T. Buzug, Computed Tomography. Springer, 2008.

[12] J. Hsieh, "Adaptive filtering approach to the streaking artifact reduction due to X-ray photon starvation," Medical Physics, vol. 25, pp. 2139_ 2147, 1998.

[13] G. L. Zeng, Medical Image Reconstruction. Springer, 2009.

[14] M. Jiang and G. Wang, "Convergence studies on iterative algorithms for image reconstruction," IEEE Transactions on Medical Imaging, vol. 22, no. 5, pp. 569-579, 2003. 
[15] L. Landweber, "An iteration formula for Fredholm integral equations of the first kind," American Journal of Mathematics, vol. 73, pp. 615-624, 1951.

[16] L. Feldkamp, L. Davis, and J. Kress, "Practical cone-beam algorithm," Journal of the Optical Society of America A, vol. 1, no. 6, pp. 612-619, 1984.

[17] V. Aurich and J. Weule, "Non-linear Gaussian filters performing edge preserving diffusion," Proc. DAGM-Symposium Mustererkennung, vol. 17 , pp. $538-545,1995$

[18] C. Tomasi and R. Manduchi, "Bilateral filtering for gray and color images," in Proc. 6th IEEE ICCV, 1998, pp. 839-846.

[19] A. M. Mendrik, E. Vonken, B. van Ginneken, H. W. de Jong, A. Riordan, T. van Seeters, E. J. Smit, M. A. Viergever, and M. Prokop, "TIPS bilateral noise reduction in 4D CT perfusion scans produces high-quality cerebral blood flow maps," Physics in Medicine and Biology, vol. 56, no. 13 , pp. 3857-3872, 2011.

[20] M. Elad, "On the origin of the bilateral filter and ways to improve it," IEEE Trans Img Proc, vol. 11, pp. 1141-1151, 2002.

[21] M. Unser, "Splines: a perfect fit for signal and image processing," IEEE Signal Processing Magazine, vol. 16, no. 6, pp. 22-38, 1999.

[22] D. L. Parker, "Optimal short scan convolution reconstruction for fan beam CT," Medical Physics, vol. 9, no. 2, pp. 254-257, 1982.

[23] L. Shepp and B. Logan, "The Fourier reconstruction of a head section," IEEE Trans Nuclear Science, vol. 21, pp. 21-43, 1974.

[24] G. H. Golub and C. Reinsch, "Singular value decomposition and least squares solutions," Numerische Mathematik, vol. 14, pp. 403-420, 1970.

[25] H. Hudson and R. Larkin, "Accelerated image reconstruction using ordered subsets of projection data," IEEE Trans Med Imaging, vol. 13, pp. 601-609, 1994.

[26] B. Keck, H. Hofmann, H. Scherl, M. Kowarschik, and J. Hornegger, "GPU-accelerated SART reconstruction using the CUDA programming environment," in Proc. SPIE Med. Img.: Physics of Medical Imaging, vol. 7258, 2009, pp. 72 582B-1.

[27] E. Y. Sidky and X. Pan, "Image reconstruction in circular cone-beam computed tomography by constrained, total-variation minimization," Physics in Medicine and Biology, vol. 53, no. 17, pp. 4777-4807, 2008.

[28] M. T. Manhart, A. Fieselmann, and Y. Deuerling-Zheng, "Evaluation of a tight frame reconstruction algorithm for perfusion C-arm CT using a realistic dynamic brain phantom," in Proc. of the Second International Conference on Image Formation in X-Ray Computed Tomography, Salt Lake City, USA, 2012, pp. 123-126.

[29] Digital brain perfusion phantom. http://www5.cs.fau.de/data/.

[30] A. J. Riordan, M. Prokop, M. A. Viergever, J. W. Dankbaar, E. J. Smit, and H. W. A. M. de Jong, "Validation of CT brain perfusion methods using a realistic dynamic head phantom," Medical Physics, vol. 38, no. 6, pp. 3212-3221, 2011

[31] A. M. Dale, B. Fischl, and M. I. Sereno, "Cortical surface-based analysis: I. Segmentation and surface reconstruction," NeuroImage, vol. 9, no. 2, pp. 179-194, 1999.

[32] A. Fieselmann, F. Dennerlein, Y. Deuerling-Zheng, J. Boese, R. Fahrig, and J. Hornegger, "A model for filtered backprojection reconstruction, artifacts due to time-varying attenuation values in perfusion C-arm CT," Physics in Medicine and Biology, vol. 56, no. 12, pp. 3701-3717, 2011.

[33] R. Yasuda, K. Royalty, K. Pulfer, D. Consigny, and C. Strother, "CArm CT measurement of cerebral blood volume using intra-arterial injection of contrast medium: An experimental study in canines," Am J Neuroradiol, vol. 33, no. 9, pp. 1696-1701, 2012.

[34] M. Ahmed, A.S.and Zellerhoff, C. Strother, K. Pulfer, Y. DeuerlingZheng, T. Redel, K. Royalty, D. Consigny, and D. Niemann, "C-Arm CT measurement of cerebral blood volume: An experimental study in canines," AJNR, vol. 30, no. 5, pp. 917-22, 2009.

[35] L. Ritschl, F. Bergner, C. Fleischmann, and M. Kachelrieß, "Improved total variation-based CT image reconstruction applied to clinical data," Physics in Medicine and Biology, vol. 56, no. 6, pp. 1545-1561, 2011.

[36] N. Strobel, O. Meissner, J. Boese, T. Brunner, B. Heigl, M. Hoheisel, G. Lauritsch, M. Nagel, M. Pfister, E.-P. Rührnschopf, B. Scholz, B. Schreiber, M. Spahn, M. Zellerhoff, and K. Klingenbeck-Regn, Multislice CT, ser. Medical Radiology. Springer Berlin Heidelberg, 2009, ch. 3D Imaging with Flat-Detector C-Arm Systems, pp. 33-51.

[37] Y. Deuerling-Zheng, M. Lell, A. Galant, and J. Hornegger, "Motion compensation in digital subtraction angiography using graphics hardware," Computerized Medical Imaging and Graphics, vol. 30, pp. 279-289, 2006.

[38] A. Fieselmann, M. Kowarschik, A. Ganguly, J. Hornegger, and R. Fahrig, "Deconvolution-based CT and MR brain perfusion measurement: Theoretical model revisited and practical implementation details," International Journal of Biomedical Imaging, 2011, article ID 467563.
[39] L. Østergaard, R. M. Weisskoff, C. Chesler, David A. Gyldensted, and B. R. Rosen, "High resolution measurement of cerebral blood flow using intravascular tracer bolus passages. Part I: Mathematical approach and statistical analysis," Magnetic Resonance in Medicine, vol. 36, no. 5, pp. 715-725, 1996.

[40] A. Savitzky and M. J. E. Golay, "Smoothing and differentiation of data by simplified least squares procedures," Analytical Chemistry, vol. 36, no. 8 , pp. 1627-1639, 1964. 